\title{
The Swire/ Chandra Survey: The X-Ray Sources
}

\section{Citation}

Wilkes, Belinda J., Roy Kilgard, Dong-Woo Kim, Minsun Kim, Mari Polletta, Carol Lonsdale, Harding E. Smith, et al. 2009. "The Swire/ Chandra Survey: The X-Ray Sources." The Astrophysical Journal Supplement Series 185 (2) (November 17): 433-450. doi:10.1088/0067-0049/185/2/433.

\section{Published Version}

doi:10.1088/0067-0049/185/2/433

\section{Permanent link}

http://nrs.harvard.edu/urn-3:HUL.InstRepos:29921904

\section{Terms of Use}

This article was downloaded from Harvard University's DASH repository, and is made available under the terms and conditions applicable to Other Posted Material, as set forth at http:// nrs.harvard.edu/urn-3:HUL.InstRepos:dash.current.terms-of-use\#LAA

\section{Share Your Story}

The Harvard community has made this article openly available.

Please share how this access benefits you. Submit a story.

\section{Accessibility}




\title{
THE SWIRE/CHANDRA SURVEY: THE X-RAY SOURCES
}

\author{
Belinda J. Wilkes ${ }^{1}$, Roy Kilgard ${ }^{2}$, Dong-Woo Kim ${ }^{1}$, Minsun Kim ${ }^{3}$, Mari Polletta ${ }^{4}$, Carol Lonsdale ${ }^{5}$, \\ Harding E. Smith ${ }^{6}$, Jason Surace ${ }^{7}$, FraZer N. Owen $^{8}$, A. Franceschini ${ }^{9}$, Brian Siana $^{10}$, and David Shupe ${ }^{10}$ \\ ${ }^{1}$ Harvard-Smithsonian Center for Astrophysics, Cambridge, MA 02138, USA \\ ${ }^{2}$ Department of Astronomy, Wesleyan University, CT 06459, USA \\ ${ }^{3}$ Korea Astronomy and Space Science Institute (KASI), 61-1, Hwaam-dong, Yuseong-gu, Deajeon 305-348, Republic of Korea \\ ${ }^{4}$ INAF-ISAF Milano, via E. Bassini 15, Milano 20133, Italy \\ ${ }^{5}$ The National Radio Astronomy Observatory ${ }^{11}$, Charlottesville, VI 22902, USA \\ ${ }^{6}$ Center for Astrophysics and Space Science, University of California, San Diego, La Jolla, CA 92093-0424, USA \\ ${ }^{7}$ Spitzer Science Center, California Institute of Technology, 100-22, Pasadena, CA 91125, USA \\ ${ }^{8}$ National Radio Astronomy Observatory ${ }^{11}$, P.O. Box 0, Socorro, NM 87801, USA \\ ${ }^{9}$ Dipartimento Di Astronomia, Universita di Padova, Vicolo Osservatorio 5, I-35122 Padua, Italy \\ ${ }^{10}$ California Institute of Technology, MC 105-24, 1200 East California Boulevard, Pasadena, CA 91125, USA \\ Received 2009 February 18; accepted 2009 October 21; published 2009 November 17
}

\begin{abstract}
We report a moderate-depth (70 ks), contiguous $0.7 \mathrm{deg}^{2}$ Chandra survey in the Lockman Hole Field of the Spitzer/ SWIRE Legacy Survey coincident with a completed, ultra-deep VLA survey with deep optical and near-infrared imaging in-hand. The primary motivation is to distinguish starburst galaxies and active galactic nuclei (AGNs), including the significant, highly obscured $\left(\log N_{\mathrm{H}}>23\right)$ subset. Chandra has detected $775 \mathrm{X}$-ray sources to a limiting broadband $(0.3-8 \mathrm{keV})$ flux $\sim 4 \times 10^{-16} \mathrm{erg} \mathrm{cm}^{-2} \mathrm{~s}^{-1}$. We present the X-ray catalog, fluxes, hardness ratios, and multi-wavelength fluxes. The $\log N$ versus $\log S$ agrees with those of previous surveys covering similar flux ranges. The Chandra and Spitzer flux limits are well matched: 771 (99\%) of the X-ray sources have infrared (IR) or optical counterparts, and 333 have MIPS $24 \mu \mathrm{m}$ detections. There are four optical-only X-ray sources and four with no visible optical/IR counterpart. The very deep $(\sim 2.7 \mu \mathrm{Jy}$ rms $)$ VLA data yield 251 $(>4 \sigma)$ radio counterparts, $44 \%$ of the X-ray sources in the field. We confirm that the tendency for lower X-ray flux sources to be harder is primarily due to absorption. As expected, there is no correlation between observed IR and X-ray fluxes. Optically bright, type 1, and red AGNs lie in distinct regions of the IR versus X-ray flux plots, demonstrating the wide range of spectral energy distributions in this sample and providing the potential for classification/source selection. Many optically bright sources, which lie outside the AGN region in the optical versus X-ray plots $\left(f_{r} / f_{x}>10\right)$, lie inside the region predicted for red AGNs in IR versus X-ray plots, consistent with the presence of an active nucleus. More than $40 \%$ of the X-ray sources in the VLA field are radio-loud using the classical definition, $R_{L}$. The majority of these are red and relatively faint in the optical so that the use of $R_{L}$ to select those AGNs with the strongest radio emission becomes questionable. Using the $24 \mu \mathrm{m}$ to radio flux ratio $\left(q_{24}\right)$ instead results in 13 of the 147 AGNs with sufficient data being classified as radio-loud, in good agreement with the $\sim 10 \%$ expected for broad-lined AGNs based on optical surveys. We conclude that $q_{24}$ is a more reliable indicator of radio-loudness. Use of $R_{L}$ should be confined to the optically selected type 1 AGN.
\end{abstract}

Key words: catalogs - quasars: general - surveys - X-rays: galaxies

Online-only material: color figures, machine-readable tables

\section{INTRODUCTION}

SWIRE, the largest Spitzer Legacy program, is tracing the evolution of dusty, star-forming galaxies, evolved stellar populations, and AGNs as a function of environment from $z \gtrsim$ 2.5 to the present epoch. SWIRE covers six fields with a total area of $49 \mathrm{deg}^{2}$ in all seven Spitzer bands, selected from the entire IRAS/DIRBE sky as those areas with the lowest 100 $\mu \mathrm{m}$ surface brightness (which scales with $N_{\mathrm{H}}$; Schlegel et al. 1998); the Lockman Hole is one of the two best regions, having several contiguous deg ${ }^{2}$ of low $N_{\mathrm{H}}\left(\sim 7 \times 10^{19} \mathrm{~cm}^{-2}\right)$ sky. SWIRE's power comes from its large surface area, its depth, which probes the universe out to redshifts $z \gtrsim 2.5$ and over time intervals $>10 \mathrm{Gyr}$, and its sensitivity to both evolved stellar populations (IRAC: $3-8 \mu \mathrm{m}$ ) and dusty objects (starbursts and obscured AGNs; MIPS: 24, 70, and $160 \mu \mathrm{m}$ ). Thus, their

\footnotetext{
${ }^{11}$ The National Radio Astronomy Observatory is a facility of the National Science Foundation operated under cooperative agreement by Associated Universities Inc.
}

distribution relative to environment, and evolution with respect to time and to the development of structure, can be studied together. SWIRE is one of the several, on-going, medium-depth surveys that fill the gap between the deep and shallow surveys, dramatically illustrating the need for a multi-layered approach to efficiently fill the $L-z$ plane in several wave bands.

The SWIRE prime science goal is to study the structure, evolution, and environments of AGNs, starbursts, and spheroidal galaxies out to $z \gtrsim 2.5$. Key to this extensive multi-wavelength campaign is an X-ray survey deep enough to distinguish starbursts and AGNs, including the significant numbers which are highly obscured, $N_{\mathrm{H}} \gtrsim 10^{23} \mathrm{~cm}^{-2}$. We carried out a Chandra $\mathrm{X}$-ray survey in the best (in terms of Galactic extinction and absence of nearby bright sources, including no bright radio sources) extragalactic $\sim 1 \mathrm{deg}^{2}$ field within SWIRE. The Xray observations cover $0.7 \mathrm{deg}^{2}$ contiguously. The broadband $(0.3-8.0 \mathrm{keV})$ flux limit is $4 \times 10^{-16} \mathrm{erg} \mathrm{cm}^{-2} \mathrm{~s}^{-1}$ at the field centers, sufficient to detect all SWIRE infrared (IR) AGNs except for those with high absorption at low redshift $(z \leqslant 0.8$, 
$\left.\log N_{\mathrm{H}} \geqslant 24\right)$ while IR galaxies are not detected. Thus we are able to distinguish all but the most highly obscured AGNs from amongst the IR sources by their X-ray emission.

The standard AGN model includes a supermassive black hole surrounded by an accretion disk in the center of a galaxy. The central regions of the AGN produce strong hard X-ray emission (and relativistic jets in radio-loud sources) along with thermal emission from an accretion disk. Gas in the vicinity is heated by the nuclear emission and produces the broad and narrow emission lines characteristic of type 1 and type 2 AGNs, respectively. The viewing orientation of an AGN affects its classification, as demonstrated by the detection of polarized broad lines in NGC1068 (Antonucci \& Miller 1985). This result led to a general acceptance that some fraction of type 2 AGNs are edge-on type $1 \mathrm{~s}$. Such unification schemes require highly opaque dust surrounding the accretion disk. In addition to obscuring our view to the nucleus, this dust is heated by the nuclear source producing strong IR emission. Thus, the observed spectral energy distribution (SED) of an AGN is dependent on the viewing angle, and the traditional optical/ultraviolet surveys are incomplete to obscured sources (e.g., Polletta et al. 2006). It is now clear that there is a significant obscured AGN population that has been missed from earlier surveys. The powerful combination of radio, X-ray, and IR observations available for the current surveys facilitates a different and potentially unbiased view of the AGN in the field and so probes the full AGN population, including obscured sources. The variety of new techniques has resulted in new types of AGN being found, including: red AGN (Cutri et al. 2002), X-ray bright optically-normal galaxies (XBONGS, Comastri et al. 2002), type 2 quasars (Norman et al. 2002), X-ray-detected Extremely Red Objects (V/EROs; Alexander et al. 2002; Brusa et al. 2005).

Despite several multi-wavelength surveys in progress during this era of Great Observatories, a full view of the AGN population remains elusive. Shallow surveys, which are dominated by type 1 AGN, find largely distinct subsets of the population depending on the wave band (Hickox et al. 2009). While overlap is significant in deeper surveys, selection in any single wave band does not find all the AGNs (Barmby et al. 2006; Polletta et al. 2006; Donley et al. 2008; Park et al. 2008). Samples of the obscured AGN remain relatively small, and biases are still in the process of being understood, so that the nature of new AGNs and their significance to the population as a whole remain undetermined. Compton-thick AGNs are hard to find because their $\mathrm{X}$-ray flux is obscured to energies $\gtrsim 10 \mathrm{keV}$. Estimates of the fraction of the general population that are Compton thick vary. X-ray-selected AGNs include $\sim 30 \%$ (Polletta et al. 2006; Treister et al. 2004) Compton-thick sources. This number doubles to $\sim 66 \%$ when IR-selected AGNs are included (Polletta et al. 2006; Treister et al. 2009). Estimates generated by stacking Xrays from IR-selected AGNs also suggest a factor of 2 increase in IR-selected compared with X-ray-selected Compton-thick AGNs (Fiore et al. 2008; Daddi et al. 2007), although the absolute numbers differ by factors of $\sim 100$ depending on selection techniques and assumptions. The latest estimate from modeling the Cosmic X-ray Background (CXRB) postulates an obscured population larger than the unobscured by a factor of 8 at low luminosities, half of which are Compton thick. This decreases to a factor of 2 at high luminosities (Gilli et al. 2007). Alternatively, many X-ray-selected, low-luminosity AGNs may be intrinsically X-ray hard rather than obscured, possibly reducing the fraction of obscured (including Compton-thick) AGNs to $\sim 20 \%$ (Hopkins et al. 2009).
A number of wide area, multi-wavelength surveys with sufficient depth to view the full AGN population are currently in progress, including: ECDFS (Lehmer et al. 2005, $0.3 \mathrm{deg}^{2}$ ), OPTX (Trouille et al. 2008, $1 \mathrm{deg}^{2}$ non-contiguous), SWIRE/ Chandra (this paper, $0.7 \mathrm{deg}^{2}$ ), AEGIS-X (Laird et al. 2009, $0.67 \mathrm{deg}^{2}$ ), and C-COSMOS (Elvis et al. 2009, 0.5+0.4 deg ${ }^{2}$ ). They are beginning to provide significant samples of the relatively rare sources, such as the high-redshift or obscured AGNs, found in small numbers in the deep Chandra surveys (Luo et al. 2008; Alexander et al. 2003). These various surveys will allow us to properly characterize these populations and understand their significance to AGNs as a whole.

In this paper, we present the $0.7 \mathrm{deg}^{2} \mathrm{SWIRE} /$ Chandra X-ray source catalog, SWIRE identifications, and IR, optical, and radio fluxes. IR properties of X-ray sources range from the powerlaw shape characteristic of AGN-dominated sources, mostly unobscured type $1 \mathrm{AGNs}$, to SEDs that are dominated by star formation or host-galaxy emission, implying obscuration of the AGN at these wavelengths (Franceschini et al. 2005; Barmby et al. 2006; Polletta et al. 2007; Feruglio et al. 2008; Cardamone et al. 2008; Gorjian et al. 2008). X-ray hardness is loosely related to the optical/IR colors, generally supporting this view. We characterize the X-ray and multi-wavelength properties of the SWIRE/Chandra sample and take an initial look at these properties as a function of X-ray hardness and radio-loudness. Detailed study and modeling of the SEDs will be presented in a companion paper (M. Polletta et al. 2010, in preparation).

\section{X-RAY DATA AND ANALYSIS}

We have obtained Chandra ACIS-I observations in a $3 \times 3$ raster of a contiguous $0.7 \mathrm{deg}^{2}$ area of sky within the Lockman Hole region of the SWIRE survey; see image in Figure 1 (left), color coded for X-ray energy. In addition to the standard 4 ACIS-I chips, we also include the ACIS-S2 chip in our analysis. The exposure time for each observation was $\sim 70 \mathrm{ks}$, reaching broadband fluxes of $\sim 4 \times 10^{-16} \mathrm{erg} \mathrm{cm}^{-2} \mathrm{~s}^{-1}$ (for $\sim$ five counts, on-axis). The nine observations, listed in Table 1 along with the Galactic $N_{\mathrm{H}}$ values from Stark et al. (1992), were obtained between 2004 September 12 and 26. They were arranged in a $3 \times 3$ raster spaced by $15^{\prime}$ in order to ensure overlap of the individual pointings. The observations were made within a 15day period with roll angles (Table 1) from 17.60-21.20, a sufficiently small range that the fields overlapped and coverage was contiguous (Figure 1, right).

The data were processed using the XPIPE pipeline developed for analysis of Chandra data for the ChaMP using CIAO v3.1 (Kim et al. 2004, hereafter DK04). XPIPE screens bad data, corrects instrumental effects remaining after the standard pipeline processing, detects the X-ray sources (using wavdetect in the broad band $0.3-8 \mathrm{keV}$ ), and determines source properties including counts, fluxes, hardness ratios, and colors using the soft $(0.3-2.5 \mathrm{keV})$, hard $(2.5-8.0 \mathrm{keV})$, and broad $(0.3-8.0 \mathrm{keV})$ bands as well as the commonly used soft $(0.5-2.0 \mathrm{keV})$ and hard (2-8 keV; Table 2). A threshold of $10^{-6}$ in wavdetect was used to accept a source, corresponding to $<1$ spurious source per ACISI chip, or four per field. To avoid finding spurious sources located at the edge of the CCD chips, a minimum of $10 \%$ of the on-axis exposure was required for source detection. Exposure maps of the SWIRE/Chandra fields were generated for each CCD at an energy of $1.5 \mathrm{keV}$ with an appropriate aspect histogram. ${ }^{12}$ The maximum value of the exposure maps is $\sim 600 \mathrm{~cm}^{2}$ counts/ photon in each CCD. Since the area with exposure map values

\footnotetext{
12 See http://cxc.harvard.edu/ciao/threads/expmap_acis_single.
} 

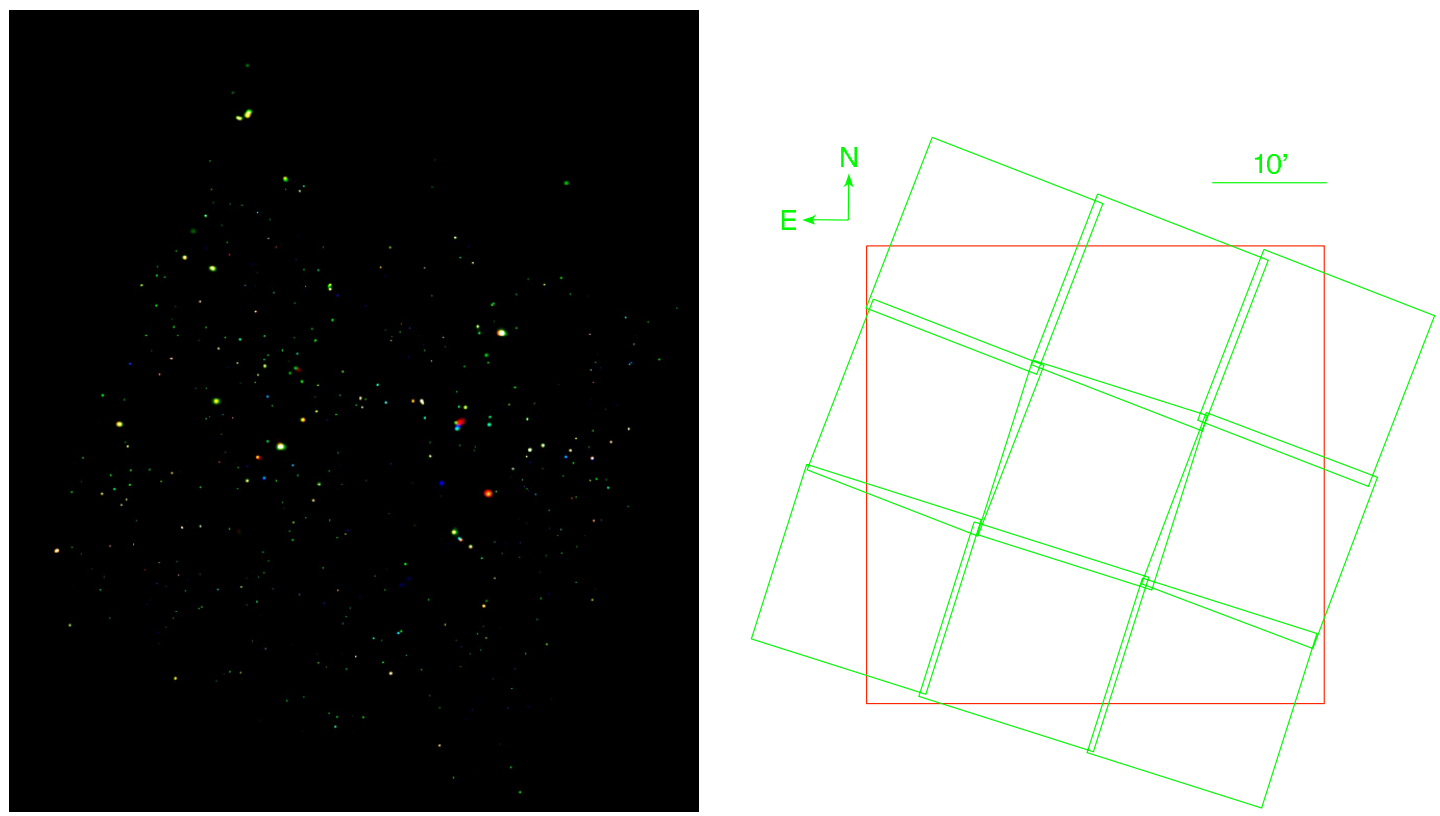

Figure 1. Left: image of the full, mosaic'd X-ray field with counts color coded by energy as follows: red (0.3-0.9 keV), green ( $0.9-2.5 \mathrm{keV})$, and blue (2.5-8 keV) so that soft sources are red and hard ones are blue. Note that sources appearing extended are generally at higher off-axis angles where the spatial resolution is lower; right: fields of view of the Chandra observations (green) and the deepest VLA data (red).

Table 1

X-ray Observations of SWIRE/Chandra Fields

\begin{tabular}{lccccc}
\hline \hline Obs. ID & $\begin{array}{c}\text { Aimpoint } \\
\text { J2000.0 }\end{array}$ & $\begin{array}{c}\text { Date Obs. } \\
\text { UT }\end{array}$ & $\begin{array}{c}\text { Exp. time } \\
(\mathrm{s})\end{array}$ & $\begin{array}{c}\text { Galactic } N_{\mathrm{H}} \\
\left(10^{19} \mathrm{~cm}^{-2}\right)\end{array}$ & \begin{tabular}{c} 
Roll Angle \\
\hline 5023
\end{tabular} \\
5024 & $10: 46: 02.15+59: 00: 58.42$ & 2004 Sep 12, 21:31:59 & 67146.56 & 6.6 & 17.60 \\
5025 & $10: 44: 48.27+58: 41: 53.88$ & 2004 Sep 16, 06:54:50 & 66297.89 & 6.4 & 17.60 \\
5026 & $10: 46: 41.57+58: 46: 49.66$ & 2004 Sep 17, 20:31:07 & 69257.73 & 6.3 & 17.60 \\
5027 & $10: 48: 34.91+58: 51: 45.76$ & 2004 Sep 18, 16:18:16 & 68863.14 & 6.4 & 17.60 \\
5028 & $10: 44: 08.82+58: 56: 04.42$ & 2004 Sep 20, 14:41:38 & 67076.31 & 6.7 & 20.07 \\
5029 & $10: 47: 55.60+59: 05: 56.46$ & 2004 Sep 23, 03:37:16 & 71145.48 & 6.5 & 21.20 \\
5030 & $10: 43: 29.40+59: 10: 14.55$ & 2004 Sep 24, 03:44:19 & 71127.09 & 7.1 & 21.20 \\
5031 & $10: 45: 22.74+59: 15: 10.64$ & 2004 Sep 25, 19:48:12 & 65748.49 & 6.9 & 21.20 \\
\hline
\end{tabular}

Table 2

Definition of Energy Bands and X-ray Colors

\begin{tabular}{lc}
\hline \hline \multicolumn{1}{c}{ Band } & Definition \\
\hline Broad $(B)$ & $0.3-8 \mathrm{keV}$ \\
Soft $(\mathrm{S})$ & $0.3-2.5 \mathrm{keV}$ \\
Hard $(\mathrm{H})$ & $2.5-8 \mathrm{keV}$ \\
Soft1 $\left(\mathrm{S}_{1}\right)$ & $0.3-0.9 \mathrm{keV}$ \\
Soft2 $\left(\mathrm{S}_{2}\right)$ & $0.9-2.5 \mathrm{keV}$ \\
\hline Conventional broad $(\mathrm{Bc})$ & $0.5-8 \mathrm{keV}$ \\
Conventional soft $(\mathrm{Sc})$ & $0.5-2 \mathrm{keV}$ \\
Conventional hard $(\mathrm{Hc})$ & $2-8 \mathrm{keV}$ \\
\hline Hardness ratio $(\mathrm{HR})$ & $(\mathrm{Hc}-\mathrm{Sc}) /(\mathrm{Hc}+\mathrm{Sc})$ \\
\hline
\end{tabular}

$<10 \%$ of the maximum in each CCD is $\sim 1 \%$ of the geometrical area of each CCD, $\sim 1 \%$ of the total number of detected sources met this criterion, assuming the sources are randomly distributed across the CCD.

A total of 897 sources were detected in this initial analysis. From this list, 80 sources were multiply-detected "duplicate" sources found in the regions of overlap between observations. In these cases, we excluded detections with lower source significance from the catalog; given that the sources are likely not variable on the timescale between observations, the lower significance is either due to larger off-axis angle, lower net counts due to telescope dither when close to a chip edge, or a combination of the two effects. An additional 42 sources were deemed to be spurious based on visual inspection of the $\mathrm{X}$-ray, optical, and IR images, consistent with the wavdetect threshold of $\sim$ four spurious sources per field. The final list (Table 3) thus contains 775 unique X-ray sources, 763 of which are found in the contiguous field with the remaining 12 detected on the $\mathrm{S} 2$ chips in the three most northerly pointings. The $\mathrm{X}$ ray sources, including positions, counts, fluxes, and hardness ratios are listed in Tables 3 and $4 .{ }^{13}$ Hardness ratios (defined as $(\mathrm{Hc}-\mathrm{Sc}) /(\mathrm{Hc}+\mathrm{Sc})$, where $\mathrm{Hc}$ and $\mathrm{Sc}$ are the counts in the hard (2.0-8.0 keV) and soft (0.5-2.0 keV) bands, respectively) were determined with a Bayesian approach using the BEHR program. ${ }^{14}$ This method is designed for low count sources where a Poisson distribution is appropriate and uses as input the hard and soft source and background counts. It was applied as described in Kim et al. (2007a) to estimate the hardness ratio and its error for all sources detected in the broad band. To estimate the flux of the X-ray point sources, we first calculated the energy conversion factor (ECF), which converts the count

\footnotetext{
13 We present a page of each table here and the full tables in the online version of the journal and on our website (http://swire.ipac.caltech.edu/swire/ swire.html). SWIRE/Chandra database access with a search capability is available at http://cosmosdb.iasf-milano.inaf.it/CHANDRA-SWIRE/index.jsp. 14 See http://hea-www.harvard.edu/AstroStat/BEHR (Park et al. 2006).
} 
Table 3

SWIRE X-ray Sources

\begin{tabular}{|c|c|c|c|c|c|}
\hline Name & $\begin{array}{l}\text { R.A. } \\
\text { J2000 }\end{array}$ & $\begin{array}{l}\text { Decl. } \\
\text { J2000 }\end{array}$ & $\begin{array}{l}\text { Pos. err }{ }^{a} \\
(\operatorname{arcsec})\end{array}$ & $\begin{array}{c}\text { X-Ray Counts } \\
(0.3-8.0 \mathrm{keV}) \\
\end{array}$ & $\begin{array}{l}\text { Count Rate } \\
\left(\text { counts s }^{-1}\right)\end{array}$ \\
\hline CXOSW J104629.3+585941 & $10: 46: 29.27$ & $58: 59: 41.3$ & 0.16 & $131.3 \pm 12.6$ & $1.96 E-03 \pm 1.88 E-04$ \\
\hline CXOSW J104616.4+585921 & $10: 46: 16.41$ & $58: 59: 21.1$ & 0.24 & $27.9 \pm 6.6$ & $4.16 E-04 \pm 9.76 E-05$ \\
\hline CXOSW J104613.5+585941 & $10: 46: 13.47$ & $58: 59: 41.6$ & 0.09 & $150.6 \pm 13.4$ & $2.24 E-03 \pm 2.00 E-04$ \\
\hline CXOSW J104647.0+585618 & $10: 46: 46.95$ & $58: 56: 18.3$ & 0.49 & $106.1 \pm 12.0$ & $1.58 E-03 \pm 1.79 E-04$ \\
\hline CXOSW J104638.5+585642 & 10:46:38.47 & $58: 56: 42.9$ & 0.51 & $50.5 \pm 8.8$ & $7.53 E-04 \pm 1.30 E-04$ \\
\hline CXOSW J104633.2+585815 & $10: 46: 33.18$ & $58: 58: 15.9$ & 0.26 & $91.4 \pm 10.8$ & $1.36 E-03 \pm 1.61 E-04$ \\
\hline CXOSW J104622.4+590052 & $10: 46: 22.40$ & $59: 00: 52.7$ & 0.26 & $25.9 \pm 6.3$ & $3.85 E-04 \pm 9.35 E-05$ \\
\hline CXOSW J104622.0+585630 & $10: 46: 21.97$ & $58: 56: 30.1$ & 0.31 & $80.4 \pm 10.3$ & $1.20 E-03 \pm 1.53 E-04$ \\
\hline CXOSW J104658.9+585959 & $10: 46: 58.91$ & $58: 59: 59.6$ & 0.83 & $33.6 \pm 7.9$ & $5.00 E-04 \pm 1.17 E-04$ \\
\hline CXOSW J104649.5+585532 & $10: 46: 49.49$ & $58: 55: 32.2$ & 0.86 & $45.1 \pm 9.2$ & $6.71 E-04 \pm 1.37 E-04$ \\
\hline CXOSW J104647.3+590122 & $10: 46: 47.30$ & $59: 01: 22.4$ & 0.49 & $67.5 \pm 9.7$ & $1.01 E-03 \pm 1.44 E-04$ \\
\hline CXOSW J104644.1+590028 & $10: 46: 44.07$ & $59: 00: 28.3$ & 0.49 & $33.1 \pm 7.2$ & $4.94 E-04 \pm 1.07 E-04$ \\
\hline CXOSW J104637.5+585914 & $10: 46: 37.49$ & $58: 59: 14.8$ & 0.65 & $21.9 \pm 6.1$ & $3.26 E-04 \pm 9.07 E-05$ \\
\hline CXOSW J104707.3+590014 & $10: 47: 07.27$ & 59:00:14.6 & 0.85 & $49.8 \pm 9.5$ & $7.42 E-04 \pm 1.41 E-04$ \\
\hline CXOSW J104654.6+585804 & $10: 46: 54.57$ & $58: 58: 04.3$ & 0.75 & $36.1 \pm 8.2$ & $5.38 E-04 \pm 1.22 E-04$ \\
\hline CXOSW J104653.3+585735 & $10: 46: 53.32$ & $58: 57: 35.2$ & 0.92 & $21.0 \pm 7.2$ & $3.13 E-04 \pm 1.07 E-04$ \\
\hline CXOSW J104651.0+585824 & $10: 46: 50.98$ & $58: 58: 24.9$ & 1.00 & $11.2 \pm 5.9$ & $1.66 E-04 \pm 8.83 E-05$ \\
\hline CXOSW J104648.1+590103 & $10: 46: 48.12$ & 59:01:03.0 & 1.08 & $16.6 \pm 6.0$ & $2.47 E-04 \pm 8.95 E-05$ \\
\hline CXOSW J104705.3+585850 & 10:47:05.26 & $58: 58: 50.4$ & 1.59 & $16.2 \pm 7.1$ & $2.42 E-04 \pm 1.06 E-04$ \\
\hline CXOSW J104703.1+585622 & $10: 47: 03.05$ & $58: 56: 22.3$ & 1.72 & $20.3 \pm 8.0$ & $3.02 E-04 \pm 1.19 E-04$ \\
\hline CXOSW J104658.4+585914 & $10: 46: 58.37$ & $58: 59: 14.7$ & 1.56 & $22.9 \pm 7.0$ & $3.41 E-04 \pm 1.05 E-04$ \\
\hline CXOSW J104632.7+585409 & $10: 46: 32.71$ & $58: 54: 09.4$ & 1.63 & $26.5 \pm 7.8$ & $3.94 E-04 \pm 1.15 E-04$ \\
\hline CXOSW J104611.1+585517 & $10: 46: 11.06$ & $58: 55: 17.5$ & 0.44 & $58.4 \pm 9.1$ & $8.69 E-04 \pm 1.36 E-04$ \\
\hline CXOSW J104607.6+585602 & 10:46:07.60 & $58: 56: 02.8$ & 0.63 & $15.6 \pm 5.5$ & $2.33 E-04 \pm 8.13 E-05$ \\
\hline CXOSW J104557.0+590000 & $10: 45: 57.00$ & 59:00:00.4 & 0.19 & $23.6 \pm 6.1$ & $3.52 E-04 \pm 9.06 E-05$ \\
\hline
\end{tabular}

Note. ${ }^{\text {a }}$ The position error was determined as described in MK07.

(This table is available in its entirety in a machine-readable form in the online journal. A portion is shown here for guidance regarding its form and content.)

rate to the flux, assuming a single power-law model for the $\mathrm{X}$ ray source spectrum with photon indices of $\Gamma=1.2,1.4$, and 1.7 (see Section 3.2.4 of Kim et al. 2007a (hereafter MK07) for a detailed description of estimating ECF). The fluxes presented in Table 4 assume $\Gamma=1.7$. For soft/hard-band fluxes where the net counts were negative, no flux estimation is provided. The $\log N-\log S$ relation (Section 4) was derived adopting the ECFs for photon indices of $\Gamma=1.4$ and 1.7 .

The X-ray flux limit of the sample is $F(0.3-8 \mathrm{keV})$ $\sim 4 \times 10^{-16} \mathrm{erg} \mathrm{cm}^{-2} \mathrm{~s}^{-1}$ (five counts, on-axis). The brightest source is at $3 \times 10^{-13} \mathrm{erg} \mathrm{cm}^{-2} \mathrm{~s}^{-1}$. This survey covers the flux range in between the deepest Chandra surveys and the widearea shallow surveys. Figure 2 shows the X-ray hardness ratio as a function of flux in the broad band. The usual trend for hard sources to have fainter fluxes is visible. Figure 3 repeats Figure 2 as a function of the hard (left) and soft (right) band fluxes. The trend is stronger in the soft band and largely absent in the hard band, consistent with the general interpretation of the hardness being due to obscuration (DK04, MK07). Radio-loud, radiointermediate, and radio-quiet sources are also labeled, based on the original $R_{L}$ classification scheme (see Section 5.5 for more discussion). Radio-loudness does not show any particular trend in these figures.

During the final stages of preparation of this paper, an $\mathrm{X}$-ray source list derived from these Chandra data was published (CLANS; Trouille et al. 2008). This source list includes 761 $\mathrm{X}$-ray sources, similar to our 775 , but a detailed comparison yields significant differences. The CLANS list was derived using a wavdetect threshold of $10^{-7}$. We find 94 CLANS sources with no match in our X-ray source list. Ten of these were in our preliminary list but were deleted as spurious following visual inspection. There are 113 sources in our X-ray list which have no CLANS match, including 33 sources with $\alpha>162.2$, a sky region not included in their analysis. With the exception of these 33, the sources which appear in only one list are at low flux levels. There are 662 sources in common, five of which are counted as double in the CLANS list but confirmed by visual inspection to be single in ours. The derived X-ray fluxes in the two samples generally agree well, except at low count rates in the hard band.

\subsection{X-ray Spectral Analysis}

Fifteen of the X-ray sources have $>300$ net counts, facilitating spectral analysis. Spectra and associated response functions were extracted and the spectra were grouped with a minimum of 15 counts per bin. Fits were performed utilizing the CIAO (v3.4) tool Sherpa with the Levenberg-Marquardt optimization method and $\chi^{2}$ statistics with variance computed from the (chidvar). $90 \%$ errors were computed using the projection method in Sherpa.

We first fit all the spectra with a power-law model $\left(F_{v}=v^{-\alpha_{e}}\right.$, where $\alpha_{E}$ is the energy index and photon index, $\left.\Gamma=1+\alpha_{e}\right)$ and cold absorption at redshift zero $\left(N_{\mathrm{H}}\right)$. The minimum allowed $N_{\mathrm{H}}$ was set to $5.9 \times 10^{19} \mathrm{~cm}^{-2}, \sim 1 \sigma$ below the lowest Galactic value (Table 1; Stark et al. 1992). The results are shown in Table 5. Eleven sources are acceptably fitted with this model alone. The average power-law slope, $\Gamma=1.89 \pm$ 0.36 , is consistent with the results for luminous sources in the Chandra Deep Field South (CDFS; Tozzi et al. 2006). For five of these 11 sources, $N_{\mathrm{H}}$ hit the minimum allowed value, suggesting a soft excess in the spectrum that was not accounted for in the model. To examine the soft excess in more detail, 
Table 4

SWIRE X-ray Sources

\begin{tabular}{|c|c|c|c|c|c|}
\hline Name & $\begin{array}{c}\text { Flux Density }{ }^{\mathrm{a}} \\
(1 \mathrm{keV})\end{array}$ & $\begin{array}{c}\text { Broadband Flux }{ }^{\mathrm{a}} \\
(0.3-8 \mathrm{keV})\end{array}$ & $\begin{array}{c}\text { Soft Flux }{ }^{\mathrm{a}} \\
(0.3-2.5 \mathrm{keV})\end{array}$ & $\begin{array}{l}\text { Hard Flux }{ }^{\mathrm{a}} \\
(2.5-8 \mathrm{keV})\end{array}$ & $\begin{array}{c}\text { Hardness } \\
\text { Ratio }^{\mathrm{b}}\end{array}$ \\
\hline CXOSW J104629.3+585941 & $5.76 \mathrm{E}-15$ & $2.24 \mathrm{E}-14$ & $1.33 \mathrm{E}-14$ & $6.45 \mathrm{E}-15$ & $-0.70_{-0.07}^{+0.05}$ \\
\hline CXOSW J104616.4+585921 & $1.23 \mathrm{E}-15$ & 4.65E-15 & $2.92 \mathrm{E}-15$ & $9.12 \mathrm{E}-16$ & $-0.75_{-0.16}^{+0.10}$ \\
\hline CXOSW J104613.5+585941 & $6.61 \mathrm{E}-15$ & $2.49 \mathrm{E}-14$ & $1.19 \mathrm{E}-14$ & $1.56 \mathrm{E}-14$ & $-0.37_{-0.08}^{+0.07}$ \\
\hline CXOSW J104647.0+585618 & 4.65E-15 & $1.91 \mathrm{E}-14$ & $1.00 \mathrm{E}-14$ & $9.40 \mathrm{E}-15$ & $-0.50_{-0.10}^{+0.09}$ \\
\hline CXOSW J104638.5+585642 & $2.22 \mathrm{E}-15$ & $8.93 \mathrm{E}-15$ & $4.50 \mathrm{E}-15$ & $4.90 \mathrm{E}-15$ & $-0.43_{-0.15}^{+0.13}$ \\
\hline CXOSW J104633.2+585815 & 4.01E-15 & $1.58 \mathrm{E}-14$ & $8.67 \mathrm{E}-15$ & $6.67 \mathrm{E}-15$ & $-0.56_{-0.10}^{+0.08}$ \\
\hline CXOSW J104622.4+590052 & $1.13 \mathrm{E}-15$ & 4.69E-15 & $1.71 \mathrm{E}-15$ & 4.44E-15 & $-0.04_{-0.20}^{+0.19}$ \\
\hline CXOSW J104622.0+585630 & $3.53 \mathrm{E}-15$ & $1.49 \mathrm{E}-14$ & $7.88 \mathrm{E}-15$ & $7.18 \mathrm{E}-15$ & $-0.50_{-0.11}^{+0.09}$ \\
\hline CXOSW J104658.9+585959 & $1.47 \mathrm{E}-15$ & $6.14 \mathrm{E}-15$ & $3.40 \mathrm{E}-15$ & $2.50 \mathrm{E}-15$ & $-0.56_{-0.19}^{+0.17}$ \\
\hline CXOSW J104649.5+585532 & $1.98 \mathrm{E}-15$ & $8.71 \mathrm{E}-15$ & $5.24 \mathrm{E}-15$ & $2.36 \mathrm{E}-15$ & $-0.69_{-0.17}^{+0.14}$ \\
\hline CXOSW J104647.3+590122 & $2.96 \mathrm{E}-15$ & $1.20 \mathrm{E}-14$ & $2.91 \mathrm{E}-15$ & $1.56 \mathrm{E}-14$ & $0.30_{-0.12}^{+0.12}$ \\
\hline CXOSW J104644.1+590028 & $1.45 \mathrm{E}-15$ & $5.84 \mathrm{E}-15$ & $2.35 \mathrm{E}-15$ & 4.90E-15 & $-0.15_{-0.18}^{+0.17}$ \\
\hline CXOSW J104637.5+585914 & $9.60 \mathrm{E}-16$ & $3.80 \mathrm{E}-15$ & $1.68 \mathrm{E}-15$ & $2.74 \mathrm{E}-15$ & $-0.25_{-0.23}^{+0.21}$ \\
\hline CXOSW J104707.3+590014 & $2.19 \mathrm{E}-15$ & $9.31 \mathrm{E}-15$ & $2.18 \mathrm{E}-15$ & $1.23 \mathrm{E}-14$ & $0.32_{-0.15}^{+0.17}$ \\
\hline CXOSW J104654.6+585804 & $1.58 \mathrm{E}-15$ & $6.83 \mathrm{E}-15$ & 4.81E-15 & $\ldots$ & $-0.87_{-0.13}^{+0.03}$ \\
\hline CXOSW J104653.3+585735 & $9.23 \mathrm{E}-16$ & $3.89 \mathrm{E}-15$ & $2.16 \mathrm{E}-15$ & $1.59 \mathrm{E}-15$ & $-0.52_{-0.29}^{+0.22}$ \\
\hline CXOSW J104651.0+585824 & $4.90 \mathrm{E}-16$ & $2.05 \mathrm{E}-15$ & $5.92 \mathrm{E}-16$ & $2.39 \mathrm{E}-15$ & $0.14_{-0.36}^{+0.40}$ \\
\hline CXOSW J104648.1+590103 & $7.29 \mathrm{E}-16$ & $2.96 \mathrm{E}-15$ & $1.31 \mathrm{E}-15$ & $2.15 \mathrm{E}-15$ & $-0.25_{-0.28}^{+0.27}$ \\
\hline CXOSW J104705.3+585850 & 7.12E-16 & $3.06 \mathrm{E}-15$ & $2.47 \mathrm{E}-15$ & $\ldots$ & $-0.78_{-0.22}^{+0.05}$ \\
\hline CXOSW J104703.1+585622 & $8.91 \mathrm{E}-16$ & $3.85 \mathrm{E}-15$ & $9.86 \mathrm{E}-16$ & 4.83E-15 & $0.23_{-0.29}^{+0.34}$ \\
\hline CXOSW J104658.4+585914 & $1.01 \mathrm{E}-15$ & 4.19E-15 & $2.41 \mathrm{E}-15$ & $1.43 \mathrm{E}-15$ & $-0.59_{-0.26}^{+0.19}$ \\
\hline CXOSW J104632.7+585409 & $1.16 \mathrm{E}-15$ & $4.90 \mathrm{E}-15$ & $1.89 \mathrm{E}-15$ & 4.34E-15 & $-0.11_{-0.24}^{+0.25}$ \\
\hline CXOSW J104611.1+585517 & $2.56 \mathrm{E}-15$ & $1.01 \mathrm{E}-14$ & $5.53 \mathrm{E}-15$ & $4.36 \mathrm{E}-15$ & $-0.55_{-0.13}^{+0.11}$ \\
\hline CXOSW J104607.6+585602 & $6.85 \mathrm{E}-16$ & $2.89 \mathrm{E}-15$ & $1.22 \mathrm{E}-15$ & $2.24 \mathrm{E}-15$ & $-0.20_{-0.27}^{+0.25}$ \\
\hline CXOSW J104557.0+590000 & $1.04 \mathrm{E}-15$ & $7.83 \mathrm{E}-15$ & 4.02E-15 & $4.11 \mathrm{E}-15$ & $-0.44_{-0.20}^{+0.16}$ \\
\hline
\end{tabular}

Notes.

${ }^{\text {a }}$ Fluxes assume Galactic $N_{\mathrm{H}}$ and $\Gamma=1.7$, in units of erg $\mathrm{cm}^{-2} \mathrm{~s}^{-1}$ (flux) and $\mathrm{erg} \mathrm{cm}^{-2} \mathrm{~s}^{-1} \mathrm{keV}^{-1}$ (flux density).

${ }^{\mathrm{b}}$ Hardness Ratio $=(\mathrm{Hc}-\mathrm{Sc}) /(\mathrm{Hc}+\mathrm{Sc})$, where $\mathrm{Hc}=$ counts in the hard band $(2.0-8 \mathrm{keV}), \mathrm{Sc}=$ Counts in the soft band $(0.5-2.0 \mathrm{keV})$, determined using a Bayesian approach which is applicable for low count sources (see the text).

(This table is available in its entirety in a machine-readable form in the online journal. A portion is shown here for guidance regarding its form and content.)

we performed fits with an absorption component frozen to the Galactic value and added a soft excess component, modeled with a power law, to the spectrum. Since the single power-law models provided statistically acceptable fits, we cannot substantially improve upon them with the additional soft component. In four cases, the additional soft component provides a visually more acceptable fit in the low energy range. For the fifth, CXOSW $\mathrm{J} 104540.1+584254$, while the single power-law fit is statistically acceptable $\left(\chi^{2}=59.50\right.$ for 50 degrees of freedom), the addition of a soft component does improve the fit. The simplest possible model is the addition of a second power-law component, which results in slopes of $\Gamma_{1}=5.00, \Gamma_{2}=1.89$, with $\chi^{2}=46.63$ for 48 degrees of freedom (see Figure 4).

Of the remaining four sources, only one, CXOSW $\mathrm{J} 104803.4+585547$, has a statistically acceptable fit to a MeweKaastra-Liedahl thermal plasma model (MEKAL) with temperature $k T=3.15 \mathrm{keV}$. It is likely that poor counting statistics prevent us from obtaining good model fits for the remaining three sources.

\subsection{Emission-line Feature in CXOSW J104404.0+590241}

Source CXOSW J104404.0+590241 shows evidence for a spectral line at $1.14 \mathrm{keV}$ (see Figure 5). It is not required for the fit, but is preferred at around the $1.5 \sigma$ level based on an $F$-test, having an (unconstrained) equivalent width $\sim 80 \mathrm{eV}$. In general, a single line is interpreted as the $\mathrm{Fe} \mathrm{K} \alpha$ line at $6.4 \mathrm{keV}$ (rest-frame), the strongest emission line present in AGN X-ray spectra. However, in this case the source would be at $z=4.6$ and the $\mathrm{X}$-ray luminosity would be very high at $4.9 \times 10^{46} \mathrm{erg} \mathrm{s}^{-1}$.

Trouille et al. (2008) give $z=0.5$ for this source (their 136) and assign a BLAGN type based on a strong Balmer series in the optical spectrum (L. Trouille 2009, private communication). The closest possible identification for the $\mathrm{X}$-ray line at this redshift is the Silicon $\mathrm{K} \alpha$ fluoresence line, rest energy $1.74 \mathrm{keV}$, yielding $z=0.52$. This line has been observed in AGNs, though not generally in isolation. For example, photoionized material in the Broad-line Radio Galaxy, 3C445, provides several emission lines, including $\mathrm{Si} \mathrm{K} \alpha$ with an equivalent width $73 \mathrm{eV}$ (Sambruna et al. 2007). At the redshift of this source, the other expected lines are redshifted to energies below the observed band in this source, apart from $\mathrm{Fe} \mathrm{K} \alpha, \sim 4.2 \mathrm{keV}$. However, the signal to noise in the higher energy X-ray spectrum is too low to confirm or exclude the presence of an $\mathrm{Fe} \mathrm{K} \alpha$ line (Figure 5). We conclude that the emission line is $\mathrm{Si} \mathrm{K} \alpha$ at $z \sim 0.5$. 


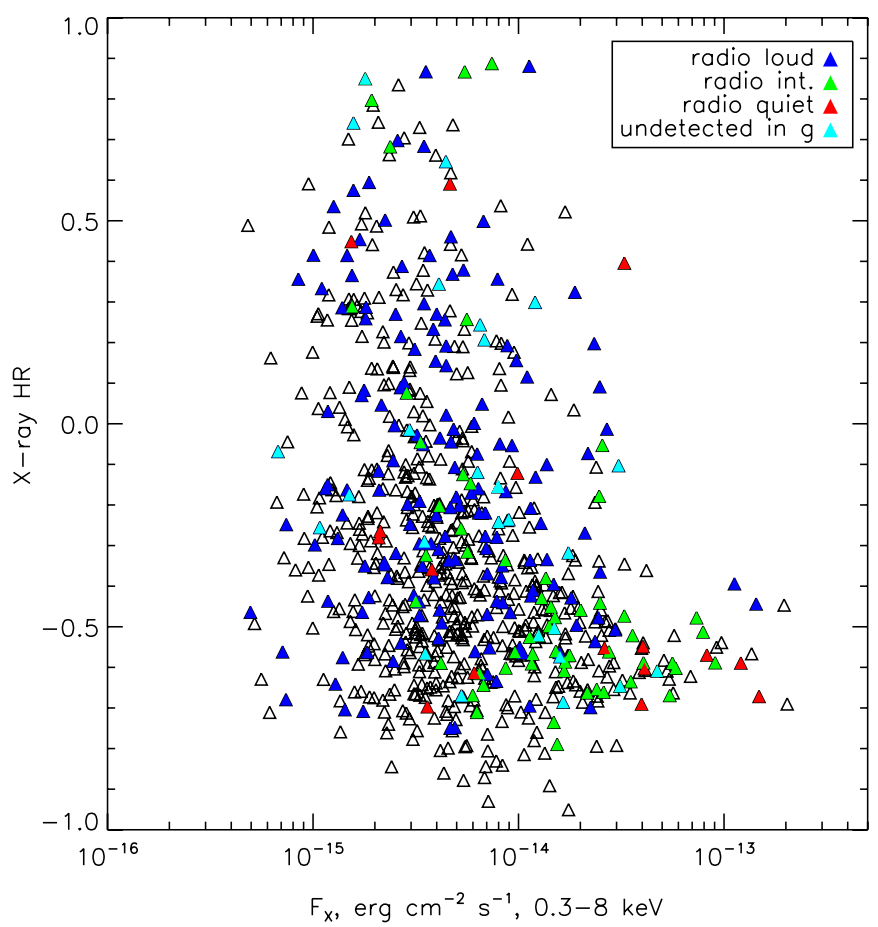

Figure 2. X-ray hardness ratio as a function of the broadband X-ray flux. The hardness ratio is determined using a Bayesian approach, which includes estimates for all targets detected in the broad band. The well-known tendency for hard sources to have fainter X-ray flux is visible. Color-coded labels show radio-loud, radio-intermediate, and radio-quiet sources, classified based on $R_{L}$ (Section 5.5). The light blue are radio-loud sources with no $g^{\prime}$ detection. The remainder (open triangles) are unobserved/undetected in the radio.

\section{MULTI-WAVELENGTH X-RAY SOURCE IDENTIFICATION}

The extensive multi-wavelength data for the SWIRE/ Chandra field are described in Polletta et al. (2006) and will

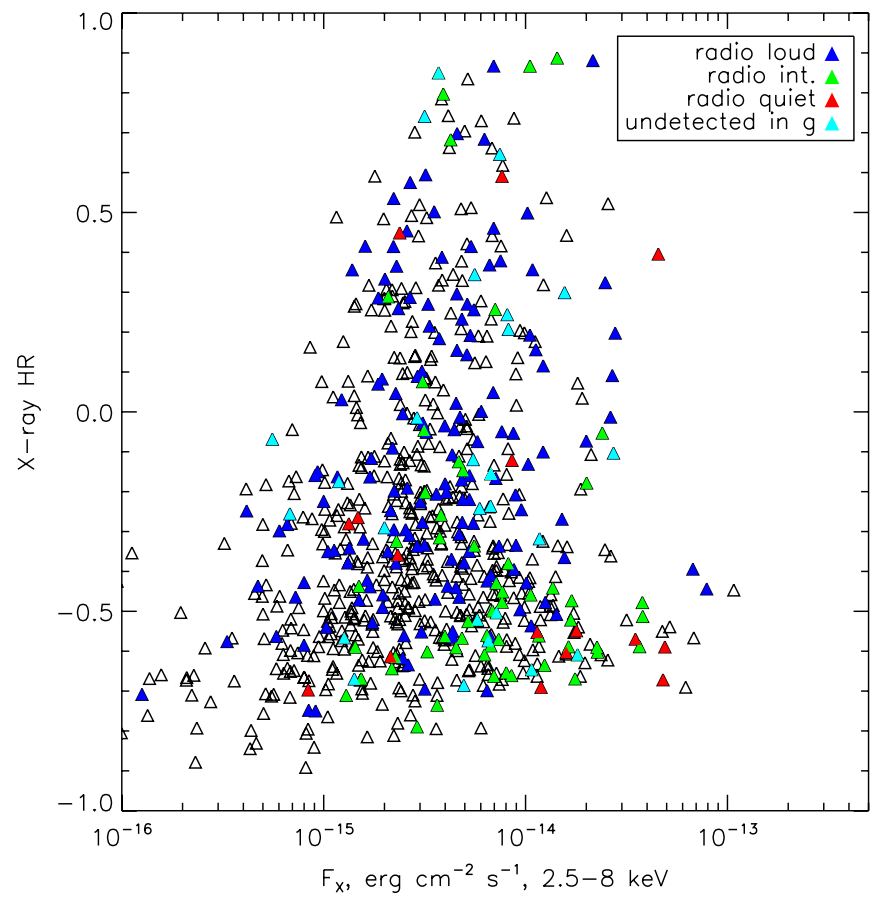

be presented in detail, along with classification of the sources and SED fitting, in a companion paper (M. Polletta et al. 2010, in preparation). For completeness, they are briefly summarized here. The Spitzer data include imaging in the 4 IRAC bands to $5 \sigma$ depths of $4.2,7.5,46$, and $47 \mu \mathrm{Jy}$ at $3.6,4.5,5.8$, and $8.0 \mu \mathrm{m}$, respectively. MIPS imaging at $24 \mu \mathrm{m}$ reaches depths of $209 \mu \mathrm{Jy}$ ( $5 \sigma$; Shupe et al. 2008). The details of the IRAC and MIPS data processing are given in Surace (2005). Here, we will refer to the optical and infrared imaging data in order to characterize the sample in comparison with other X-ray surveys. Optical imaging in $\mathrm{U}, g^{\prime}, r^{\prime}$, and $i^{\prime}$, to $5 \sigma$ limiting Vega magnitudes of 24.8, 25.9, 25.2, and 24.4, respectively, was obtained with the MOSAIC Camera on the KPNO 4 m Mayall telescope for the field center and with $\sim 0.5-1$ mag brighter limits toward the field edge (Polletta et al. 2006).

The X-ray source list was cross correlated with the optical and Spitzer source lists in search of matches. The SWIRE catalog contains all sources detected in at least one IR band in the Lockman Hole field. The cross-match procedure searches for sources within a circular radius corresponding to the geometric sum of $2^{\prime \prime}$ and the X-ray positional error or 2", whichever was greater, to ensure no potential matches were missed. We find an IR counterpart for 749 X-ray sources, and 333 have $24 \mu \mathrm{m}$ identifications. Visual inspection of the images was carried out for 68 sources with multiple associations. The closest source is preferred when it was more than $\sim 2$ times closer than any other. For counterparts at similar distances from the $\mathrm{X}$-ray source, the brightest in the IR is generally preferred. In some cases, the source SED is taken into account, preferentially selecting the counterpart with an AGN-like SED. This inspection led to the selection of preferred counterparts in 63 cases while in the remaining five cases, the identification remains ambiguous. Further inspection of the 26 sources with no IR counterpart revealed the presence of an IR source below the formal detection threshold in eight cases. In 10 cases, a visible counterpart is not listed in the catalogs due to the presence of a nearby bright

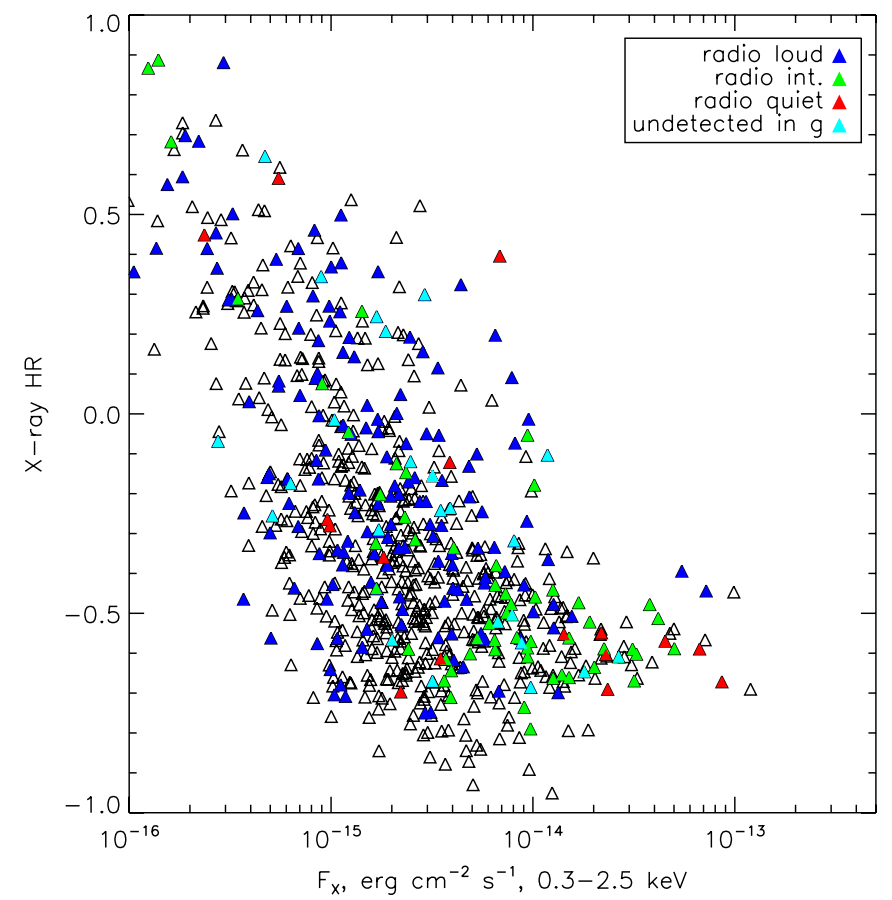

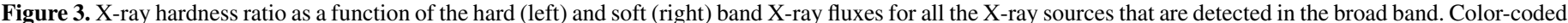

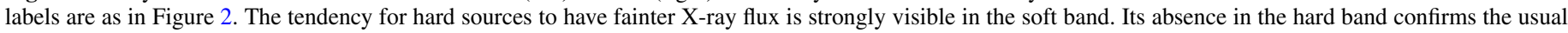
interpretation that soft X-ray absorption reduces the soft and broadband fluxes, causing harder spectra and lower observed fluxes in these sources. 
Table 5

Power-law Fits for SWIRE X-ray Sources

\begin{tabular}{|c|c|c|c|}
\hline Source & $N_{\mathrm{H}}\left(10^{20} \mathrm{~cm}^{-2}\right)$ & $\Gamma$ & $\chi^{2} / \mathrm{DOF}$ \\
\hline CXOSW J104655.5+590301 & $11.9_{-11.9}^{+17.3}$ & $1.65_{-0.33}^{+0.38}$ & $35.77 / 37$ \\
\hline CXOSW J104540.1+584254 & $0.59_{-0.0^{\mathrm{b}}}^{+3.26}$ & $2.27_{-0.22}^{+0.25}$ & $59.80 / 50$ \\
\hline CXOSW J104929.2+585338 & $0.59_{-0.0^{\mathrm{b}}}^{+6.55}$ & $2.26_{-0.14}^{+0.30}$ & $89.79 / 70$ \\
\hline CXOSW J104803.4+585547 & $4.94_{-0.0^{\mathrm{b}}}^{+25.5}$ & $2.04_{-0.38}^{+0.81}$ & $25.97 / 16$ \\
\hline CXOSW J104415.8+590101 & $0.59_{-0.0^{\mathrm{b}}}^{+17.9}$ & $1.78_{-0.21}^{+0.49}$ & $24.71 / 26$ \\
\hline CXOSW J104404.0+590241 & $9.16_{-9.16}^{+23.0}$ & $2.10_{-0.40}^{+0.62}$ & $19.58 / 25$ \\
\hline CXOSW J104343.2+585535 & $10.6_{-10.6}^{+19.7}$ & $1.75_{-0.39}^{+0.48}$ & $24.99 / 24$ \\
\hline CXOSW J104847.0+590455 & $0.59_{-0.0^{\mathrm{b}}}^{+15.8}$ & $2.26_{-0.30}^{+0.54}$ & $20.17 / 18$ \\
\hline CXOSW J104811.7+591046 & $0.59_{-0.0^{\mathrm{b}}}^{+16.0}$ & $2.09_{-0.25}^{+0.63}$ & $26.74 / 19$ \\
\hline CXOSW J104422.6+591304 & $13.0_{-13.0}^{+18.5}$ & $1.73_{-0.32}^{+0.38}$ & $53.39 / 65$ \\
\hline CXOSW J104321.3+590943 & $2.81_{-2.22^{\mathrm{b}}}^{+9.29}$ & $1.53_{-0.16}^{+0.23}$ & $67.08 / 83$ \\
\hline CXOSW J104503.4+591242 & $9.82_{-9.82}^{+20.8}$ & $1.81_{-0.36}^{+0.47}$ & $37.06 / 25$ \\
\hline CXOSW J104511.2+591625 & $7.85_{-7.85}^{+13.0}$ & $1.91_{-0.31}^{+0.38}$ & $35.02 / 40$ \\
\hline CXOSW J104743.5+591849 & $0.59_{-0.0^{\mathrm{b}}}^{+14.1}$ & $1.90_{-0.21}^{+0.45}$ & $15.49 / 23$ \\
\hline CXOSW J104713.6+591501 & $0.59_{-0.0^{\mathrm{b}}}^{+9.43}$ & $2.00_{-0.32}^{+0.46}$ & $19.44 / 17$ \\
\hline
\end{tabular}

Notes. Errors quoted are 99\% confidence intervals computed using the project function in Sherpa.

${ }^{\text {a }}$ Fit with pl+nei $\left(\Gamma=2.13, k T=0.065\right.$, reduced $\left.\chi^{2}=0.87\right)$, pl+bbody $(\Gamma=$ $2.66, k T=2.77$, reduced $\left.\chi^{2}=0.97\right)$, or 2 power laws $\left(\Gamma_{1}=5.00, \Gamma_{2}=1.89\right.$, reduced $\chi^{2}=0.85$ ).

${ }^{\mathrm{b}}$ Hard parameter space minimum.

${ }^{c}$ No good fit was found.

${ }^{\mathrm{d}}$ Fit by a MEKAL model with $k T=3.15 \mathrm{keV}$.

${ }^{\mathrm{e}}$ Shows evidence for a spectral line at $1.14 \mathrm{keV}$. It is not required for the fit, but is preferred at around the $1.5 \sigma$ level.

star. Of the remaining eight $\mathrm{X}$-ray sources, four only have optical identifications and four are genuine X-ray only sources for which the optical and IR emission is fainter than the SWIRE sensitivity limits. Thus we have identified an optical/IR counterpart for 771 X-ray sources, or $>99 \%$ of the sample. Given the uncertain IDs, contaminating sources, and very faint flux levels of some of the identifications, we have accurate optical and/or IR flux measurements for $744 \mathrm{X}$-ray sources, corresponding to $96 \%$ of the entire X-ray sample (Table 7). Those sources with no optical/IR counterpart, those with ambiguous IDs, and those with contaminating sources that prevent count extraction are labeled in Table 7 (electronic version).

A deep $1.4 \mathrm{GHz}$ radio map covering the central $40^{\prime} \times 40^{\prime}$ of the SWIRE/Chandra field was obtained at the Very Large Array (VLA). Owen \& Morrison (2008) present a 5 $\sigma$ catalog for which the flux limit in the center of the radio field is $15 \mu \mathrm{Jy}$ and a factor of $\sim 5$ brighter by $20^{\prime}$ off-axis (Owen \& Morrison 2008). Shallower data are available for the full Chandra field of view. In this paper, we use a radio source list extending down to a deeper, $4 \sigma$, flux limit, reliable given the additional requirement of identification with an X-ray source. This yields a radio flux limit $(4 \sigma)$ of $11 \mu \mathrm{Jy}$ at the field center. The radio source list was cross correlated with the X-ray source list to determine candidate radio identifications. Candidate matches were determined based upon the positional errors in both radio (which take into account extent) and X-ray and then confirmed by visual inspection. The low density of radio and $\mathrm{X}$-ray sources results in no ambiguous matches. Radio sources were identified with $251 \mathrm{X}$-ray sources in the overlapping field of view (Figure 1, right). We take a first look at the classification of the radio-detected sources in this paper. A detailed analysis of the radio properties will be presented in a future paper.

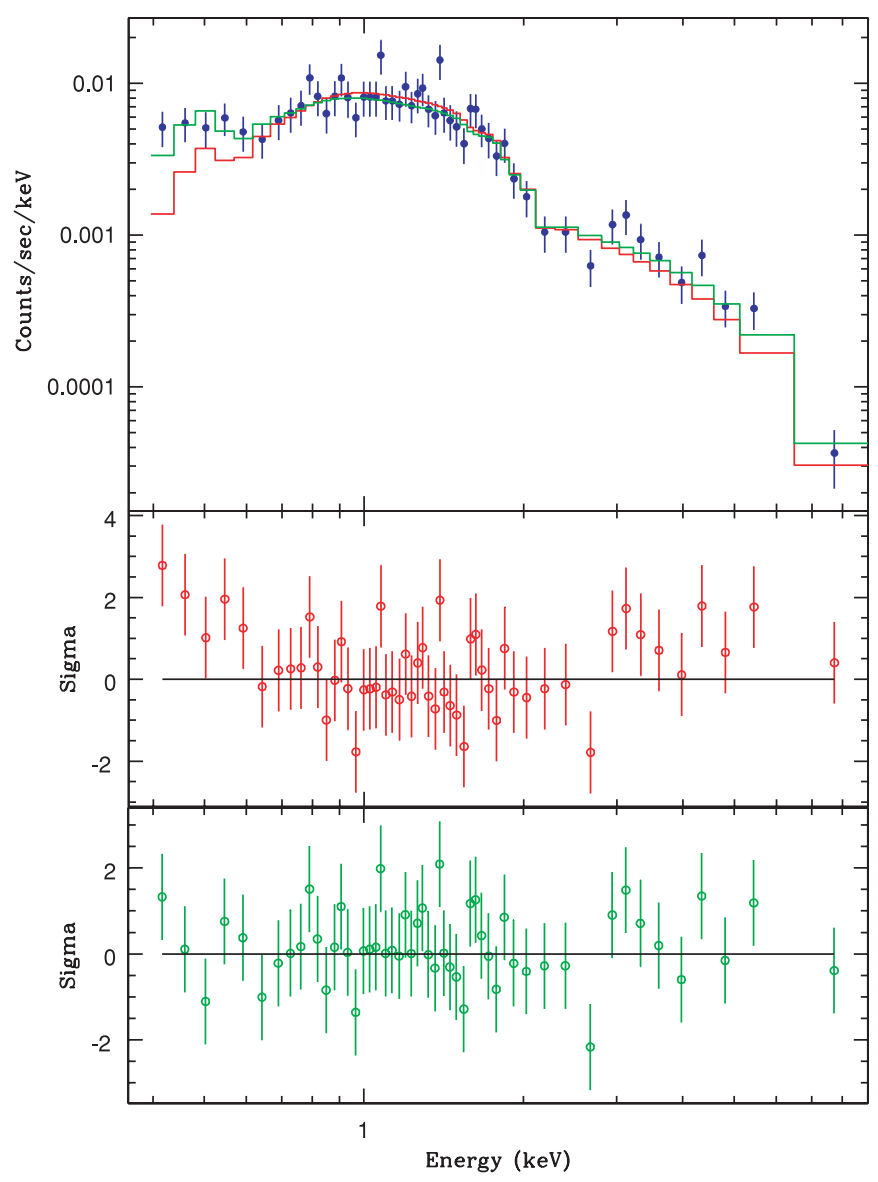

Figure 4. X-ray spectrum of the source CXOSW J104540.1+584254. Plotted in red is the best single power-law fit, and in green, a fit utilizing two power-law components, the simplest possible model to account for the clear soft excess in the spectrum. The residuals to both fits are shown underneath using the same color coding.

(A color version of this figure is available in the online journal.)

\subsection{Extended X-ray Sources}

We searched for extended X-ray sources using vtpdetect. Two candidates were found: CXOSW J104740.7+590704 and CXOSW J104653.1+592648. Modeling of their point-spread function (PSF) at $1 \mathrm{keV}$ confirmed their extent at 95\% significance.

CXOSW J104740.7+590704 appears to be associated with a group of galaxies in the optical and IR images. The central galaxy is at a redshift of $z=0.354$. The X-ray emission is soft $(\mathrm{HR}=-0.22)$ and can be fitted with a simple power law of slope $\Gamma=2.25$. The extent of the X-ray emission is almost an arcminute in radius, though the association of galaxies is smaller. The $0.3-8 \mathrm{keV}$ flux is $1.0 \times 10^{-13} \mathrm{erg} \mathrm{cm}^{-2} \mathrm{~s}^{-1}$ which, at the redshift of the central galaxy, corresponds to a luminosity of $4.5 \times 10^{43} \mathrm{erg} \mathrm{s}^{-1}$. Though the X-ray emission does not appear to contain any discrete sources, it remains possible that one or more AGNs contribute.

CXOSW J104653.1+592648 is associated with two galaxies in the IR that cannot be visually separated in the optical. The diffuse X-ray emission is concentrated in a much smaller area than the previous source: (30" in radius) which, given the source's large off-axis angle (7!3), suggests that the diffuse emission is likely associated with these two galaxies only. The $\mathrm{X}$-ray emission is quite soft $(\mathrm{HR}=-0.43)$ and the spectrum is well fitted by a soft power law with absorption and photon index, $\Gamma=3.0$. The unabsorbed X-ray flux is $1.3 \times 10^{-13} \mathrm{erg} \mathrm{cm}^{-2} \mathrm{~s}^{-1}$. 


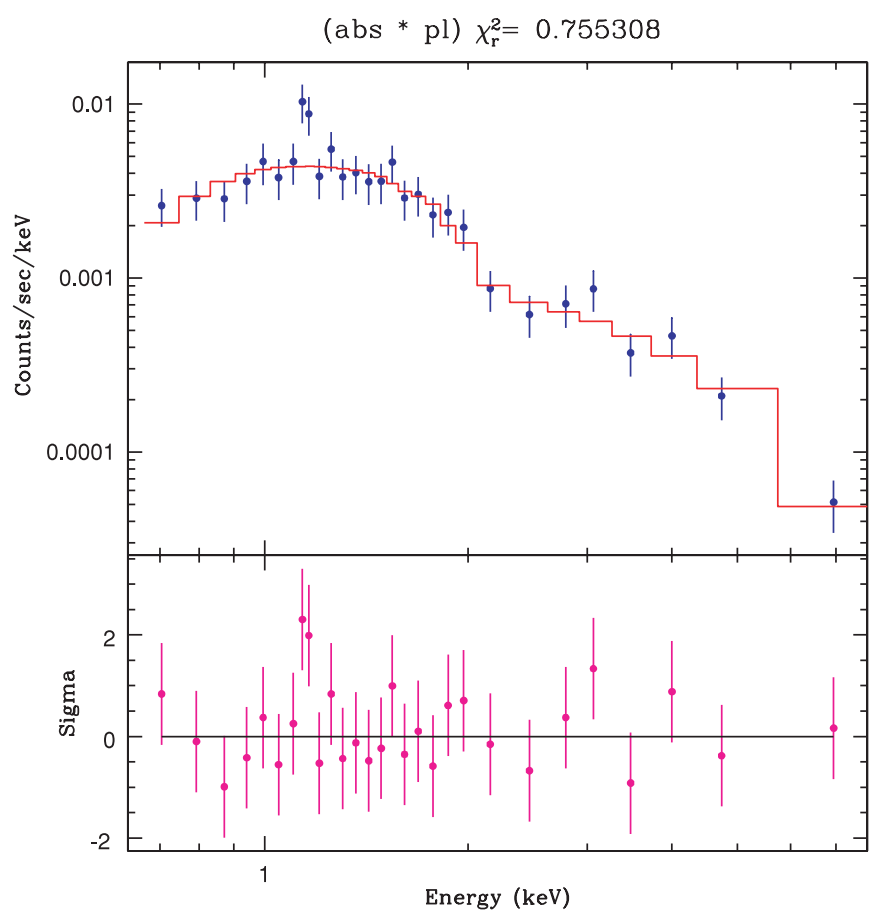

Figure 5. X-ray spectrum of the source CXOSW J104404.0+590241 with a best-fit single power-law model. The residuals are shown below. There is the suggestion of a spectral line at $\sim 1.14 \mathrm{keV}$ identified with $\mathrm{Si} \mathrm{K} \alpha$ at a redshift $\sim 0.5$. The signal to noise is too low at higher energies to confirm (or not) the presence of an $\mathrm{Fe} \mathrm{K} \alpha$ line at the same redshift, with energy $\sim 4.2 \mathrm{keV}$.

(A color version of this figure is available in the online journal.)

A spectroscopic redshift has not been measured for either IRidentified galaxy so the luminosity cannot be determined. No discrete sources are detected in the X-ray emission which is centered on the gap between the galaxies, so, though still possible, it is unlikely to be dominated by an AGN.

\section{THE $\log N$ VERSUS $\log S$ RELATION}

To determine an accurate $\log N-\log S$ relation, it is necessary to correct for the incompleteness of the sample as well as for instrumental effects such as vignetting and the off-axis degradation of the PSF. The actual source detection probability in a Chandra field is a complex function of off-axis angle and source counts: the detection probability decreases as off-axis angle increases and as source counts decrease (e.g., MK07). Therefore, to accurately determine the sky coverage of the SWIRE/Chandra sample, we performed a series of Monte Carlo simulations to correct incompleteness and biases within the sample fields. The technique for the Monte Carlo simulations is based on previous studies (e.g., Kim \& Fabbiano 2003; MK07; Kim et al. 2007b) and consists of three steps: (1) generating artificial X-ray sources with MARX, ${ }^{15}$ (2) adding them to the original observed image without subtracting any real, observed $\mathrm{X}$-ray sources, and (3) detecting these artificial sources with wavdetect and extracting source properties. In step (2), we used the real Chandra observations to accurately reflect the effects of background counts and source confusion/crowding in the SWIRE fields.

We performed simulations using all four CCDs in all nine observations (see Section 2), generating 1000 artificial X-ray sources in each of our nine sample fields extending to a flux level

\footnotetext{
15 See http://space.mit.edu/CXC/MARX/ and MARX 4.0 Technical Manual.
}

$10 \%$ of the survey detection limit. This corresponds to $\sim 13,000$ artificial X-ray sources per $\mathrm{deg}^{2}$. The number of artificial sources detected in each field depends on the effective exposure time of the observation and the neutral hydrogen column density, $N_{\mathrm{H}}$, toward the observed region of the sky.

The observed X-ray differential $\log N-\log S$ is described by a broken/double power law with a faint slope of $\sim-1.5$ and a bright slope of $\sim-2.5$ (Yang et al. 2004; Basilakos et al. 2005; Chiappetti et al. 2005) in most energy bands; however, the break flux has not been well determined. In our simulations, we assumed a cumulative $\log N-\log S$ distribution with a single power law of slope of -1 , corresponding to a slope of -2 in the differential $\log N-\log S$. This is the average of the faint and bright slopes from the literature, in the $0.3-8 \mathrm{keV}$ band. Since we use the fraction of artificial sources detected as a function of flux to determine the sensitivity (Vikhlinin et al. 1995; Kim $\&$ Fabbiano 2003), the exact form of the assumed $\log N-\log$ $S$ distribution is not critical. From the assumed $\log N-\log S$ distribution, we selected artificial source fluxes and placed them in the actual event files at random positions, taking care not to overcrowd each CCD chip (see below). Applying the same detection software to these events files as we did to the original observations, we found that, on average, $24 \%$ of the 9000 artificial X-ray sources are detected in our simulations. The flux range of the detected, artificial sources, $5 \times 10^{-16}$ to $5 \times 10^{-10} \mathrm{erg} \mathrm{cm}^{-2} \mathrm{~s}^{-1}$ in the broad band (Table 2), covers the flux range of the observed SWIRE/Chandra X-ray point sources $\left(4 \times 10^{-16}\right.$ to $\left.2 \times 10^{-13} \mathrm{erg} \mathrm{cm}^{-2} \mathrm{~s}^{-1}\right)$. The result was a total of 2,127 artificial X-ray sources in the nine SWIRE/Chandra fields. At 2.8 times the 775 observed sources, this is statistically sufficient to derive the sky coverage as a function of flux and to correct the effects of incompleteness and bias on the $\log N-\log S$ relations derived for the SWIRE/Chandra sample.

The spectrum of the artificial sources was assumed to be a power law. Observed photon indices in this flux range generally span $\Gamma=1.5-2$ (KD04, MK07). In addition, Tozzi et al. (2006) performed X-ray spectral analysis for 82 X-ray bright sources in the CDFS, and found a weighted mean value for the slope of the power-law spectrum, $\langle\Gamma\rangle \simeq 1.75 \pm 0.02$, and no significant correlation between the photon index, $\Gamma$, and the intrinsic absorption column density $N_{H \text {,int }}$. The flux range of these bright sources in the CDFS overlaps the faint flux end of the SWIRE/Chandra sources. We therefore assumed that the SWIRE/Chandra sources have a photon index, $\Gamma \sim 1.7$. We assumed a Galactic absorption, $N_{\mathrm{H}}$ (Stark et al. 1992), for each observation; we did not include intrinsic absorption for the artificial source spectra. The spectrum of each X-ray point source was generated using the $\mathrm{XSPEC}^{16}$ package.

The artificial source's position was randomly selected in each CCD chip area, but was rejected if the source area at a selected position had an exposure map value of less than $10 \%$ of the maximum. This requirement is identical to that in the SWIRE/ Chandra X-ray point-source catalog reduction procedure. To avoid overcrowding of the artificial sources, the $\sim 250$ artificial sources per CCD were divided into several groups to be added into the observed image: while we did not allow the artificial $\mathrm{X}$-ray point sources to overlap one another, we allowed overlap between artificial and real X-ray sources to provide an estimate of source confusion in each observed field. This resulted in $\sim 10$ simulated images per ACIS-I CCD, corresponding 360 CCD images (event files) on which we ran wavdetect (xapphot). Since

\footnotetext{
16 See http://xspec.gsfc.nasa.gov/.
} 

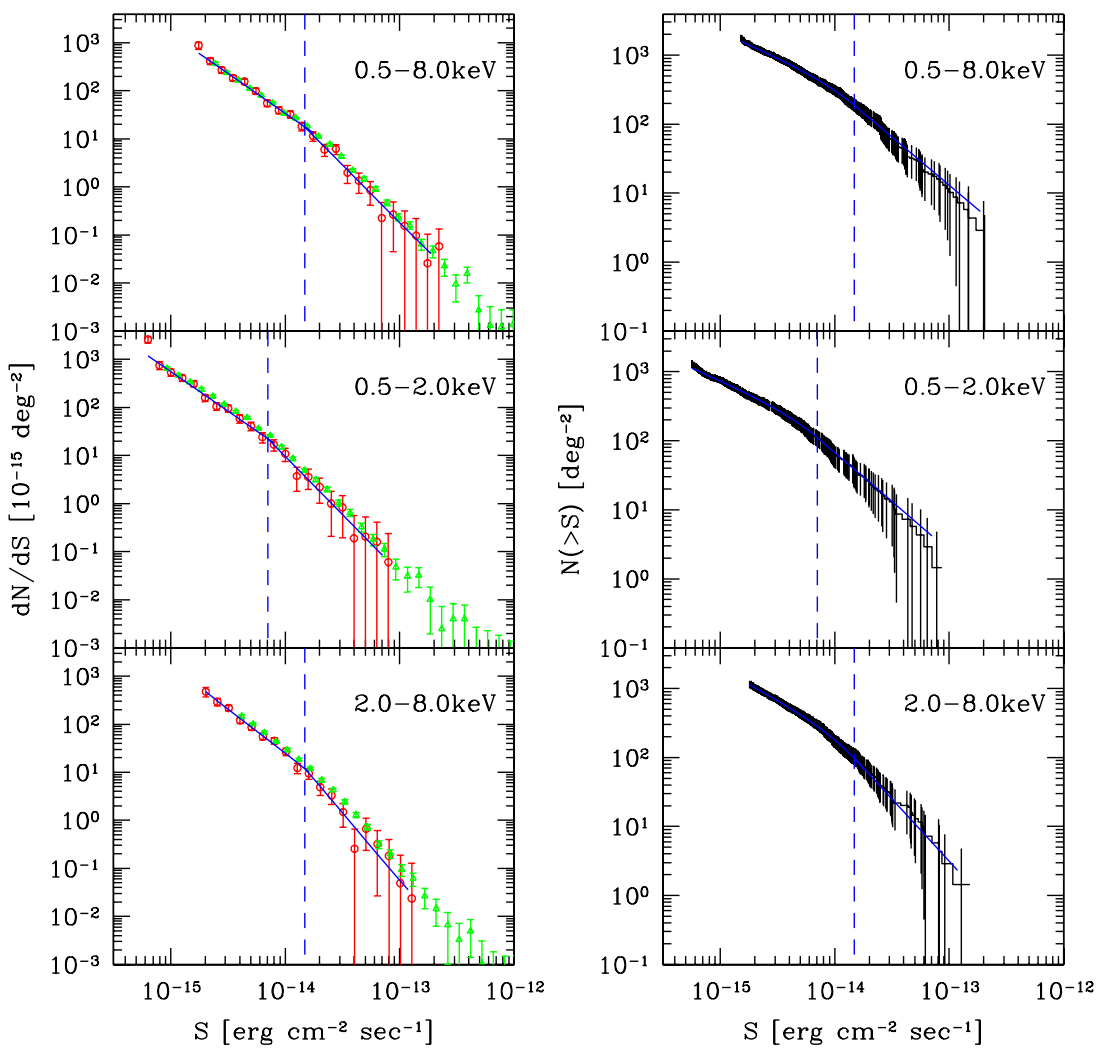

Figure 6. Differential (left) and cumulative (right) $\log N$-log $S$ distribution for the SWIRE/Chandra field (red) in comparison with those from the ChaMP full catalog (green, from Kim et al. 2007a). The blue dashed line indicates the best-fit break energy. The blue solid line indicates the best-fit broken power law.

$\sim 24 \%$ of the artificial sources $(\sim 2100)$ are detected, on average we added $\sim 6$ detectable, artificial sources to each simulated image. The net counts of those artificial sources that overlap/ blend with real sources were corrected following the methods described in Section 3.2.2 of MK07.

Using the simulation results, we derived the sky coverage as a function of flux in six energy bands (Table 2). The sky coverage area is the fraction of artificial sources detected at a given flux, multiplied by the total sky area, and it is used to correct the incompleteness and bias in the derived $\log N-\log S$ relations. The full sky area of the SWIRE/Chandra is $0.7 \mathrm{deg}^{2}$. The geometrical area of a Chandra CCD chip is $0.0196 \mathrm{deg}^{2}$; however, the net effective area is slightly larger due to the dither. To accurately calculate the effective area, we follow the same method as xapphot: all pixels in the exposure map were summed, excluding those pixels with an exposure map value less than $10 \%$ of the maximum within the corresponding source area. This criterion automatically excludes pixel positions located near the edge of the CCD chip. We note that our sample is complete at the $90 \%$ level at a flux of $1.6 \times 10^{-14} \mathrm{erg} \mathrm{cm}^{-2} \mathrm{~s}^{-1}$ in the $0.5-8 \mathrm{keV}$ band when $\Gamma=1.4$ is assumed.

The cumulative $\log N-\log S$ relation for sources brighter than a given flux $S$, corrected by the corresponding sky coverage at $S$, is

$$
N(>S)=\sum_{S_{i}>S} \frac{1}{\Omega_{i}},
$$

where $S_{i}$ is the flux of the $i$ th X-ray point source and $\Omega_{i}$ is the sky coverage, that is, the maximum solid angle over which a source with flux $S_{i}$ is detectable. Using the SWIRE/Chandra X-ray point sources and the corresponding sky coverage, we derived the cumulative $\log N-\log S$ relations for the SWIRE/Chandra
$\mathrm{X}$-ray point sources. Since the differential $\log N-\log S$ relation is a derivative form of the cumulative $\log N-\log S$ relation, we derived it from the cumulative $\log N-\log S$ relation resulting from Equation (1) as follows:

$$
\left.\frac{d N}{d S}\right|_{i}=-\frac{N_{i+1}-N_{i}}{S_{i+1}-S_{i}},
$$

where $N_{i}$ is the cumulative source number at flux $S_{i}$. Since the sky coverage rapidly decreases near the faint flux limit, there are large statistical errors for the $\log N-\log S$ distribution at faint fluxes. Thus, for better statistics, we present the $\log N-\log S$ relations brighter than the flux corresponding to $10 \%$ of the full sky coverage. In Figure 6, we display the SWIRE/ Chandra differential (left panels) and cumulative (right panels) $\log N-\log S$ relations in three energy bands. Statistical errors on the $\log N-\log S$ are assigned following Gehrels (1986). We fitted the differential $\log N-\log S$ with a broken power law as follows:

$$
\frac{d N}{d S}= \begin{cases}K\left(S / S_{\text {ref }}\right)^{-\gamma_{1}}, & S<S_{b}, \\ K\left(S_{b} / S_{\text {ref }}\right)^{\left(\gamma_{2}-\gamma_{1}\right)}\left(S / S_{\text {ref }}\right)^{-\gamma_{2}}, & S \geqslant S_{b},\end{cases}
$$

where $K$ is a normalization constant and $S_{\text {ref }}$ is a normalization flux. In this study, we set a normalization flux of $S_{\text {ref }}=$ $10^{-15} \mathrm{erg} \mathrm{cm}^{-2} \mathrm{~s}^{-1}, S_{b}$ is the break flux at which the slope of the differential $\log N-\log S$ changes, and $\gamma_{1}$ and $\gamma_{2}$ are faint and bright power-law indices. The best-fit parameters for the differential $\log N-\log S$ for various X-ray energy bands and photon indices of 1.4 and 1.7 are listed in Table 6 and displayed in Figure 6. The best-fit parameters for the differential $\log \mathrm{N}$ $\log S$ of the ChaMP and ChaMP+CDFs (Kim et al. 2007b) are 
Table 6

List of Best Fit Parameters

\begin{tabular}{|c|c|c|c|c|c|c|}
\hline DATA & $\Gamma_{p h}$ & Band $^{\mathrm{a}}$ & $K^{\mathrm{b}}$ & $\gamma_{1}^{\mathrm{c}}$ & $\gamma_{2}{ }^{\mathrm{d}}$ & $S_{b}{ }^{\mathrm{e}}$ \\
\hline \multirow[t]{12}{*}{ SWIRE } & \multirow[t]{3}{*}{1.4} & $\mathrm{~S}$ & $1011_{-86}^{+88}$ & $1.89_{-0.07}^{+0.08}$ & $2.35_{-0.30}^{+0.47}$ & $12.7_{-5.3}^{+11.5}$ \\
\hline & & $\mathrm{H}$ & $1263_{-351}^{+481}$ & $1.65_{-0.20}^{+0.21}$ & $2.49_{-0.16}^{+0.24}$ & $7.5_{-0.7}^{+2.5}$ \\
\hline & & $\mathrm{B}$ & $1540_{-351}^{+170}$ & $1.67_{-0.07}^{+0.06}$ & $2.71_{-0.53}^{+0.52}$ & $23.4_{-24.9}^{+5.2}$ \\
\hline & \multirow[t]{3}{*}{1.7} & $S$ & $974_{-85}^{+86}$ & $1.85_{-0.07}^{+0.07}$ & $2.39_{-0.32}^{+0.46}$ & $13.5_{-5.6}^{+9.2}$ \\
\hline & & $\mathrm{H}$ & $1762_{-448}^{+604}$ & $1.88_{-0.20}^{+0.20}$ & $2.47_{-0.19}^{+0.39}$ & $7.9_{-1.4}^{+5.1}$ \\
\hline & & B & $1489_{-448}^{+161}$ & $1.68_{-0.06}^{+0.06}$ & $2.70_{-0.41}^{+0.52}$ & $22.2_{-24.1}^{+4.8}$ \\
\hline & \multirow[t]{3}{*}{1.4} & $\mathrm{Sc}$ & $560_{-45}^{+45}$ & $1.65_{-0.14}^{+0.09}$ & $2.42_{-0.34}^{+0.49}$ & $7.1_{-3.2}^{+2.9}$ \\
\hline & & $\mathrm{Hc}$ & $1722_{-632}^{+322}$ & $1.84_{-0.31}^{+0.10}$ & $2.81_{-0.54}^{+0.72}$ & $14.9_{-7.6}^{+7.5}$ \\
\hline & & $\mathrm{Bc}$ & $1548_{-632}^{+247}$ & $1.66_{-0.13}^{+0.09}$ & $2.40_{-0.23}^{+0.25}$ & $14.8_{-4.7}^{+6.2}$ \\
\hline & \multirow[t]{3}{*}{1.7} & $\mathrm{Sc}$ & $579_{-48}^{+48}$ & $1.66_{-0.14}^{+0.09}$ & $2.41_{-0.33}^{+0.49}$ & $7.3_{-3.3}^{+3.1}$ \\
\hline & & $\mathrm{Hc}$ & $1384_{-83}^{+69}$ & $1.72_{-0.05}^{+0.03}$ & $2.59_{-0.23}^{+0.26}$ & $9.5_{-3.0}^{+1.9}$ \\
\hline & & $\mathrm{Bc}$ & $1384_{-83}^{+160}$ & $1.66_{-0.10}^{+0.08}$ & $2.41_{-0.23}^{+0.35}$ & $13.4_{-4.1}^{+6.1}$ \\
\hline \multirow[t]{12}{*}{ ChaMP $^{f}$} & \multirow[t]{3}{*}{1.4} & $S$ & $769_{-14}^{+14}$ & $1.57_{-0.01}^{+0.01}$ & $2.41_{-0.05}^{+0.05}$ & $9.9_{-1.6}^{+0.7}$ \\
\hline & & $\mathrm{H}$ & $1828_{-43}^{+48}$ & $1.81_{-0.01}^{+0.01}$ & $2.58_{-0.05}^{+0.05}$ & $14.2_{-1.1}^{+0.9}$ \\
\hline & & B & $1614_{-43}^{+28}$ & $1.65_{-0.01}^{+0.01}$ & $2.44_{-0.05}^{+0.06}$ & $25.0_{-1.9}^{+1.9}$ \\
\hline & \multirow[t]{3}{*}{1.7} & S & $783_{-15}^{+15}$ & $1.58_{-0.01}^{+0.01}$ & $2.42_{-0.05}^{+0.05}$ & $10.5_{-0.8}^{+0.8}$ \\
\hline & & $\mathrm{H}$ & $1774_{-41}^{+44}$ & $1.80_{-0.01}^{+0.01}$ & $2.58_{-0.05}^{+0.05}$ & $13.5_{-0.9}^{+0.9}$ \\
\hline & & B & $1505_{-41}^{+25}$ & $1.65_{-0.01}^{+0.01}$ & $2.45_{-0.05}^{+0.06}$ & $21.9_{-1.7}^{+1.7}$ \\
\hline & \multirow[t]{3}{*}{1.4} & $\mathrm{Sc}$ & $607_{-12}^{+12}$ & $1.54_{-0.02}^{+0.02}$ & $2.36_{-0.05}^{+0.05}$ & $6.8_{-0.5}^{+0.5}$ \\
\hline & & $\mathrm{Hc}$ & $2040_{-50}^{+50}$ & $1.82_{-0.01}^{+0.01}$ & $2.65_{-0.07}^{+0.07}$ & $19.2_{-1.8}^{+6.3}$ \\
\hline & & $\mathrm{Bc}$ & $1557_{-50}^{+28}$ & $1.64_{-0.01}^{+0.01}$ & $2.48_{-0.05}^{+0.05}$ & $22.9_{-1.6}^{+1.6}$ \\
\hline & \multirow[t]{3}{*}{1.7} & $\mathrm{Sc}$ & $612_{-12}^{+12}$ & $1.53_{-0.02}^{+0.02}$ & $2.36_{-0.04}^{+0.05}$ & $6.7_{-0.5}^{+0.5}$ \\
\hline & & $\mathrm{Hc}$ & $1932_{-48}^{+46}$ & $1.82_{-0.01}^{+0.01}$ & $2.64_{-0.07}^{+0.07}$ & $17.8_{-1.7}^{+4.4}$ \\
\hline & & $\mathrm{Bc}$ & $1407_{-48}^{+25}$ & $1.64_{-0.01}^{+0.01}$ & $2.48_{-0.05}^{+0.05}$ & $19.2_{-1.4}^{+1.3}$ \\
\hline \multirow{2}{*}{$\begin{array}{l}\mathrm{ChaMP}^{\mathrm{f}}{ }^{\mathrm{f}}{ }^{\mathrm{a}}{ }^{\mathrm{a}}\end{array}$} & \multirow[t]{2}{*}{1.4} & $\mathrm{Sc}$ & $571_{-11}^{+11}$ & $1.49_{-0.02}^{+0.02}$ & $2.36_{-0.05}^{+0.05}$ & $6.5_{-0.4}^{+0.4}$ \\
\hline & & $\mathrm{Hc}$ & $1258_{-29}^{+29}$ & $1.58_{-0.01}^{+0.01}$ & $2.59_{-0.05}^{+0.06}$ & $14.4_{-0.9}^{-0.4}$ \\
\hline
\end{tabular}

Notes.

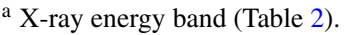

b Normalization constant.

${ }^{\mathrm{c}}$ Faint power index of a broken power law.

${ }^{\mathrm{d}}$ Bright power index of a broken power law.

e Break flux in units of $10^{-15} \mathrm{erg} \mathrm{cm}^{-2} \mathrm{~s}^{-1}$.

${ }^{\mathrm{f}}$ From Kim et al. 2007b.

also listed in Table 6 for ease of comparison. The choice of the photon index, $\Gamma$, has little effect on $\gamma_{1}$ and $\gamma_{2}$, but it shifts $S_{b}$ somewhat. In the different energy bands, the break flux, $\mathrm{S}_{b}$, has different values, similar to the results of the ChaMP and the ChaMP+CDFs. We note that the best-fit parameters $\gamma_{1}$ and $\gamma_{2}$ and $S_{b}$ agree, within the uncertainties, with previous studies covering comparable flux ranges, such as the ChaMP (Kim et al. 2007b). The faint end slope is somewhat steeper than that for the combined ChaMP+CDFs (Table 6) or the results of Trouille et al. (2008). This is most likely due to the inclusion of the fainter flux range from the CDFs in the latter, combined surveys.

\section{MULTI-WAVELENGTH PROPERTIES OF THE X-RAY SOURCES}

In Table 7, we present multi-wavelength fluxes for the SWIRE/Chandra X-ray sources along with the SWIRE ID number for the matched source. We include $g^{\prime}, r^{\prime}$ optical magnitudes, $3.6 \mu \mathrm{m}$ and $8.0 \mu \mathrm{m}$ Spitzer IRAC fluxes, $24 \mu \mathrm{m}$ Spitzer MIPS fluxes, and $20 \mathrm{~cm}(1.4 \mathrm{GHz})$ VLA radio fluxes. The full table is available in electronic form only.

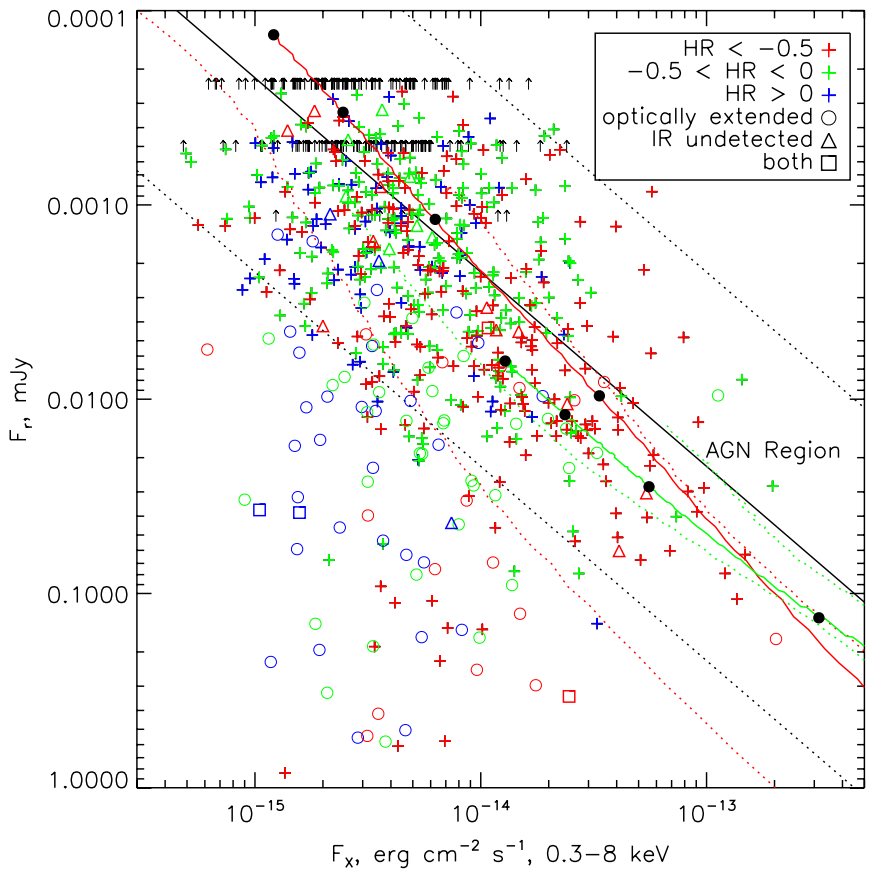

Figure 7. Optical $r^{\prime}$ band as a function of broadband X-ray flux for the SWIRE $\mathrm{X}$-ray sources. The black solid and dotted lines show $f_{x} / f_{r}=0.1,1,10$, providing a guide to where extragalactic sources (mostly AGNs) generally lie in this figure ("AGN region," Stocke et al. 1991). Optically extended and IR undetected sources are indicated by different symbols as listed, with squares indicating those which are both. X-ray hard (HR $>0)$, intermediate $(-0.5<$ $\mathrm{HR}<0)$, and soft $(\mathrm{HR}<-0.5)$ sources are labeled by color. Tracks for a median $( \pm 90 \%)$ AGN SED (Elvis et al. 1994) and a median red ( $\pm 90 \%)$ AGN SED (Kuraszkiewicz et al. 2009b) as a function of redshift (up to $z=4$ ) are displayed as green and red solid (dotted) lines, respectively (see Section 5.1). Black dots indicate redshifts of $1,2,3$, and 4 from the lower right. Optical upper limits (indicating the $90 \%$ completeness level) are shown as black arrows with the different levels indicating different optical data sets.

\subsection{X-ray and Optical Properties}

Figure 7 shows $r^{\prime}$ magnitude versus broadband X-ray flux for the SWIRE/Chandra X-ray sample. The $f_{x} / f_{r}=0.1-10$ region defined to include most extragalactic sources (most of which are AGNs) in the Einstein Medium Sensitivity Survey (EMSS, hereafter "the AGN region; Stocke et al. 1991) is shown by black dashed lines. X-ray sources identified with extended optical sources and those with no IR counterpart are labeled. Sources are colored according to their X-ray hardness ratios: hard (blue), medium (green), and soft (red). A hardness ratio, $\mathrm{HR} \sim 0$, implies an equivalent hydrogen absorption column density, $N_{\mathrm{H}} \sim 10^{22} \mathrm{~cm}^{-2}$, for a typical AGN power-law spectrum $(z=0)$.

Figure 7 also includes tracks for optically bright, type 1 (Elvis et al. 1994, green lines) and near-IR red (Kuraszkiewicz et al. 2009b, red lines) AGN SEDs, based on these low-redshift $(z \lesssim$ 0.4 ) samples, as a function of redshift up to $z=4$ (at the lower flux end). The $90 \%$ envelopes, shown as dotted lines, include the range in shape around this median, but do not include the range in luminosity expected at a given redshift. The tracks for the AGN SEDs are computed over a redshift range from $z=0.025$ to $z=4$ in increments of $z=0.025$. At each redshift interval, the luminosity distance corresponding to the redshift is computed. This distance is used to compute the observed flux for an object at that redshift in each of the bands plotted. No absorption is assumed. For the X-ray flux, we use a monochromatic flux density at $1 \mathrm{keV}$ assuming a power-law spectrum with a slope 
Table 7

SWIRE X-ray Sources: Multi-wavelength Fluxes ${ }^{\mathrm{a}}$

\begin{tabular}{|c|c|c|c|c|c|c|c|c|}
\hline Name & SWIRE ID & $\begin{array}{l}\Delta \text { pos. }^{\mathrm{b}} \\
(\operatorname{arcsec})\end{array}$ & $r^{\prime} \mathrm{mag}$ & $g^{\prime} \mathrm{mag}$ & $\begin{array}{c}3.6 \mu \mathrm{m} \text { Flux } \\
(\mu \mathrm{Jy})\end{array}$ & $\begin{array}{c}8 \mu \mathrm{m} \text { Flux } \\
(\mu \mathrm{Jy})\end{array}$ & $\begin{array}{c}24 \mu \mathrm{m} \text { Flux } \\
(\mu \mathrm{Jy})\end{array}$ & $\begin{array}{c}20 \mathrm{~cm} \text { flux } \\
(\mu \mathrm{Jy})\end{array}$ \\
\hline CXOSW J104629.3+585941 & 561274 & 0.26 & $24.43 \pm 0.09$ & $24.89 \pm 0.09$ & $11.86 \pm 0.45$ & $30.50 \pm 3.18$ & $-209.0 \pm 0.00$ & $13.6 \pm 3.4$ \\
\hline CXOSW J104616.4+585921 & 559904 & 0.87 & $22.16 \pm 0.02$ & $24.00 \pm 0.07$ & $15.33 \pm 0.53$ & $-40.00 \pm 0.00$ & $-209.0 \pm 0.00$ & $39.5 \pm 7.6$ \\
\hline CXOSW J104613.5+585941 & 559874 & 0.25 & $22.97 \pm 0.03$ & $23.34 \pm 0.06$ & $26.57 \pm 0.66$ & $127.34 \pm 3.61$ & 1147. \pm 17.0 & $92.0 \pm 5.5$ \\
\hline CXOSW J104647.0+585618 & 560498 & 0.94 & $24.61 \pm 0.11$ & $25.15 \pm 0.11$ & $6.49 \pm 0.40$ & $47.76 \pm 3.43$ & $-209.0 \pm 0.00$ & $23.0 \pm 4.2$ \\
\hline CXOSW J104638.5+585642 & 560041 & 0.44 & $-25.20 \pm 0.00$ & $-25.90 \pm 0.00$ & $25.96 \pm 0.63$ & $65.64 \pm 3.08$ & $193.6 \pm 17.3$ & $-14.7 \pm 0.0$ \\
\hline CXOSW J104633.2+585815 & 560612 & 0.94 & $20.49 \pm 0.02$ & $21.90 \pm 0.02$ & $217.91 \pm 1.81$ & $95.33 \pm 5.67$ & 1188. \pm 19.3 & $266.0 \pm 8.2$ \\
\hline CXOSW J104622.4+590052 & 561464 & 0.50 & $-25.20 \pm 0.00$ & $-25.90 \pm 0.00$ & $4.87 \pm 0.50$ & $-40.00 \pm 0.00$ & $-209.0 \pm 0.00$ & $15.7 \pm 3.9$ \\
\hline CXOSW J104622.0+585630 & 558585 & 0.31 & $18.45 \pm 0.02$ & $19.76 \pm 0.06$ & $265.98 \pm 1.85$ & $272.25 \pm 5.80$ & $750.9 \pm 18.1$ & $421.2 \pm 3.3$ \\
\hline CXOSW J104658.9+585959 & 564107 & 0.88 & $-25.20 \pm 0.00$ & $-25.90 \pm 0.00$ & $10.88 \pm 0.44$ & $-40.00 \pm 0.00$ & $164.9 \pm 16.6$ & $23.0 \pm 3.8$ \\
\hline CXOSW J104649.5+585532 & 560200 & 0.98 & $22.00 \pm 0.03$ & $22.65 \pm 0.03$ & $25.08 \pm 0.57$ & $32.19 \pm 3.06$ & $-209.0 \pm 0.00$ & $-16.0 \pm 0.0$ \\
\hline CXOSW J104647.3+590122 & 564038 & 2.57 & $23.94 \pm 0.06$ & $24.30 \pm 0.07$ & $22.85 \pm 0.51$ & $39.14 \pm 3.29$ & $312.1 \pm 16.9$ & $93.0 \pm 6.1$ \\
\hline CXOSW J104644.1+590028 & 563124 & 1.63 & $22.59 \pm 0.04$ & $23.52 \pm 0.05$ & $29.87 \pm 0.67$ & $78.35 \pm 3.42$ & $803.9 \pm 17.5$ & $29.9 \pm 5.6$ \\
\hline CXOSW J104637.5+585914 & 561669 & 1.56 & $23.39 \pm 0.04$ & $24.43 \pm 0.08$ & $33.64 \pm 0.68$ & $-40.00 \pm 0.00$ & $294.6 \pm 18.9$ & $-13.2 \pm 0.0$ \\
\hline CXOSW J104707.3+590014 & 564944 & 1.53 & $21.51 \pm 0.02$ & $23.03 \pm 0.06$ & $28.77 \pm 0.65$ & $27.37 \pm 3.23$ & $-209.0 \pm 0.00$ & $-19.6 \pm 0.0$ \\
\hline CXOSW J104654.6+585804 & 562426 & 0.85 & $22.91 \pm 0.03$ & $23.60 \pm 0.06$ & $16.62 \pm 0.52$ & $-40.00 \pm 0.00$ & $-209.0 \pm 0.00$ & $-14.7 \pm 0.0$ \\
\hline CXOSW J104653.3+585735 & 561949 & 1.15 & $23.49 \pm 0.05$ & $24.78 \pm 0.09$ & $24.81 \pm 0.70$ & $-40.00 \pm 0.00$ & $-209.0 \pm 0.00$ & $-14.7 \pm 0.0$ \\
\hline CXOSW J104651.0+585824 & 562326 & 1.34 & $23.47 \pm 0.04$ & $24.40 \pm 0.08$ & $42.93 \pm 0.77$ & $43.02 \pm 3.27$ & $-209.0 \pm 0.00$ & $-14.7 \pm 0.0$ \\
\hline CXOSW J104648.1+590103 & 563893 & 1.43 & $-25.20 \pm 0.00$ & $25.53 \pm 0.14$ & $8.44 \pm 0.44$ & $-40.00 \pm 0.00$ & $-209.0 \pm 0.00$ & $17.0 \pm 4.0$ \\
\hline CXOSW J104705.3+585850 & 563861 & 4.30 & $24.28 \pm 0.08$ & $25.21 \pm 0.11$ & $23.18 \pm 0.55$ & $60.23 \pm 3.47$ & $375.6 \pm 19.0$ & $-16.0 \pm 0.0$ \\
\hline CXOSW J104703.1+585622 & 561941 & 3.17 & $24.08 \pm 0.07$ & $24.80 \pm 0.09$ & $26.34 \pm 0.62$ & $37.50 \pm 3.28$ & $299.7 \pm 17.0$ & $21.9 \pm 4.5$ \\
\hline CXOSW J104658.4+585914 & 563547 & 2.69 & $21.95 \pm 0.02$ & $22.85 \pm 0.06$ & $57.27 \pm 0.82$ & $-40.00 \pm 0.00$ & $-209.0 \pm 0.00$ & $28.2 \pm 6.7$ \\
\hline CXOSW J104632.7+585409 & 557958 & 1.59 & $-25.20 \pm 0.00$ & $-25.90 \pm 0.00$ & $5.66 \pm 0.39$ & $-40.00 \pm 0.00$ & $-209.0 \pm 0.00$ & $32.6 \pm 4.0$ \\
\hline CXOSW J104611.1+585517 & 556975 & 0.60 & $23.69 \pm 0.05$ & $24.10 \pm 0.07$ & $10.42 \pm 0.52$ & $-40.00 \pm 0.00$ & $-209.0 \pm 0.00$ & $88.4 \pm 3.4$ \\
\hline CXOSW J104607.6+585602 & 557127 & 1.58 & $-25.20 \pm 0.00$ & $-25.90 \pm 0.00$ & $12.61 \pm 0.50$ & $-40.00 \pm 0.00$ & $-209.0 \pm 0.00$ & $256.0 \pm 3.2$ \\
\hline CXOSW J104557.0+590000 & 558749 & 0.35 & $23.28 \pm 0.04$ & $24.42 \pm 0.08$ & $8.46 \pm 0.49$ & $51.00 \pm 3.69$ & $-209.0 \pm 0.00$ & $47.1 \pm 5.5$ \\
\hline
\end{tabular}

\section{Notes.}

${ }^{a}$ Negative fluxes indicate a $4 \sigma$ upper limit for the radio and $5 \sigma$ for Spitzer and optical fluxes.

b Distance between X-ray and SWIRE sources.

(This table is available in its entirety in a machine-readable form in the online journal. A portion is shown here for guidance regarding its form and content.)

of $\Gamma=1.7$. The $1 \mathrm{keV}$ luminosities $(\log )$ of the two medians are 44.61 (Elvis et al. 1994) and 43.51 (Kuraszkiewicz et al. 2009b). We smoothed the Elvis et al. (1994) $\pm 90 \%$ envelopes slightly in the radio range to remove artifacts due to poor data sampling.

As is typical, most of the X-ray sources lie within the range expected for extragalactic sources, which are predominantly AGNs $\left(f_{x} / f_{r}=0.1-10\right.$, "AGN region"). Below this region, where $f_{x} / f_{r}<0.1$, there are 102 sources, $13 \%$ of the sample. The Elvis et al. (1994) median as a function of redshift (green lines) predicts AGNs in a small area at the bright end of the AGN region in Figure 7, demonstrating the particular subset of the luminous AGN population targeted in traditional blue, optical surveys. The red AGN median (Kuraszkiewicz et al. 2009b, red lines) covers a much broader part of the AGN region, extending to fainter fluxes (Figure 7) and suggesting that faint sources are predominantly red and/or high redshift. Red optical colors may be due to a combination of one or more factors including: Eddington ratio $\left(L / L_{\mathrm{Edd}}\right)$, obscuration, hostgalaxy, and scattered light contributions (Kuraszkiewicz et al. 2009a). A decrease in $L / L_{\mathrm{Edd}}$ decreases the strength of the optical-UV blue bump (Witt et al. 1997) so that sources with low $L / L_{\text {Edd }}$ will tend to lie on the optically fainter side of the AGN region (Kuraszkiewicz et al. 2009a). Pure obscuration of optically bright, type 1 AGN moves them below the AGN region in Figure 7 into the range of galaxies and XBONGS. Thus X-ray hard sources in this region may be low-redshift, obscured AGNs.

The few X-ray sources with no Spitzer detection (Section 3) are distributed throughout Figure 7 and so are likely a mixture of source types.

\subsection{X-ray Hardness Ratio}

The X-ray hardness ratio distribution in Figure 7 generally supports the above discussion. The tendency for hard sources to lie at fainter soft X-ray flux is generally interpreted as due to obscuration (DK04, MK07, Section 2). At higher redshift, though, the effect of obscuration at low energies is shifted out of the observed band, softening the observed spectrum so that obscured, higher redshift sources cannot be distinguished by their X-ray hardness. Thus, sources that are observed as $\mathrm{X}$-ray hard tend to lie at low redshift (MK07). An investigation of the relation between optical/IR SEDs and X-ray obscuration requires redshifts in order to place reasonable limits on the amount of X-ray absorption for each source and will be presented in the companion SED paper (M. Polletta et al. 2010, in preparation).

Sources at faint flux levels in Figure 7 are preferentially hard. Most of the soft sources lie in the AGN region. Those sources that are optically bright and X-ray faint lie below the AGN region in Figure 7, are X-ray medium/hard and optically extended, consistent with being low-redshift galaxies or obscured, type 1 AGNs.

\subsection{X-ray and Mid-IR Properties}

Figure 8 shows the broadband X-ray versus $3.6 \mu \mathrm{m}$, $4.5 \mu \mathrm{m}, 5.8 \mu \mathrm{m}$, and $8.0 \mu \mathrm{m}$ fluxes with the tracks for median optically selected, type 1 (green) and red (red) AGN SEDs superposed. Optically extended X-ray sources generally lie at higher IR fluxes than the AGN in these figures, due to the lower observed X-ray flux of galaxies or low-luminosity/absorbed 

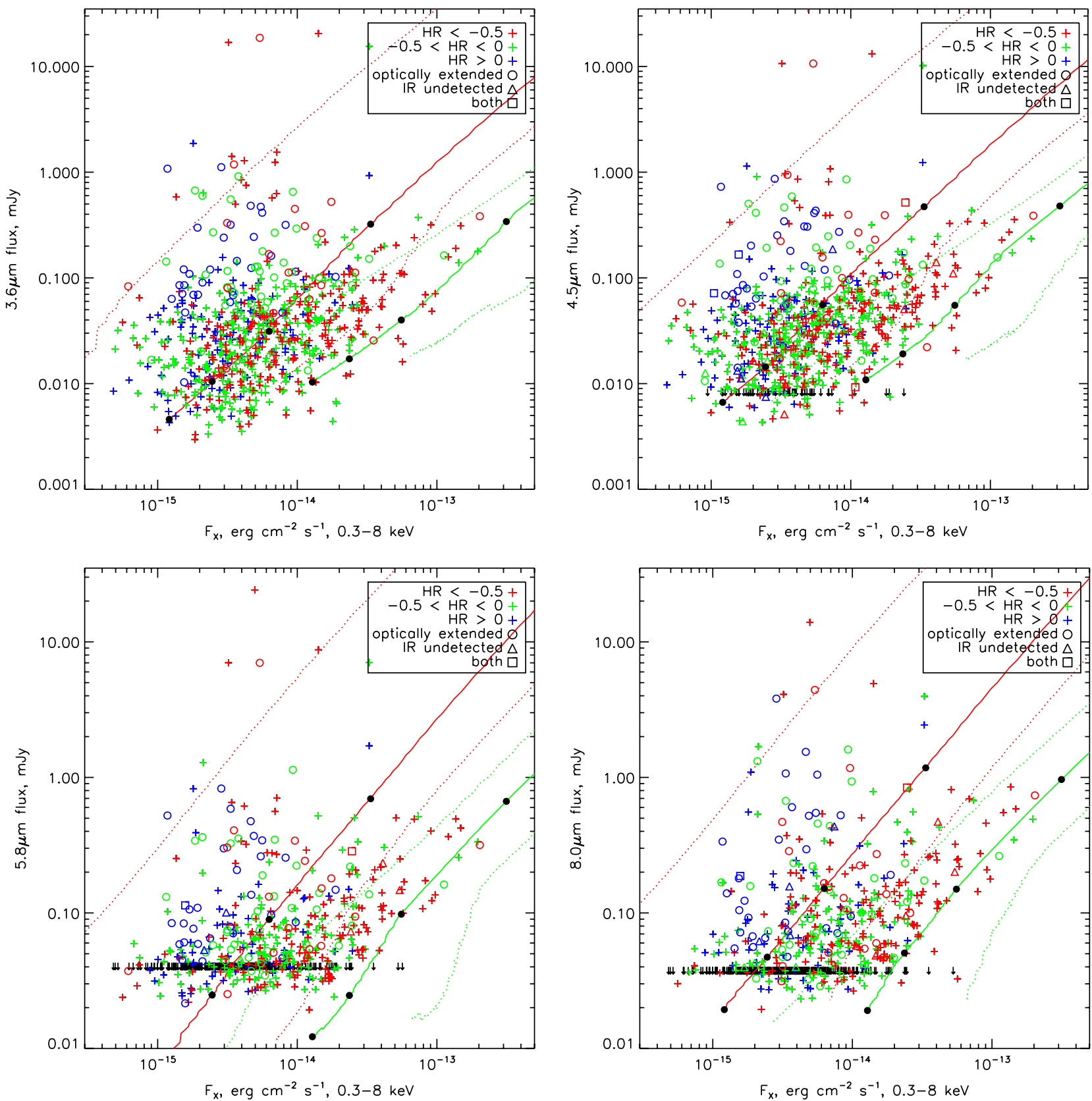

Figure 8. Spitzer IR as a function of broadband X-ray flux for the SWIRE X-ray sources in each IRAC wave band. Symbols and lines are as in Figure 7. Upper limits $(5 \sigma)$ are indicated by black arrows. The tracks for optically selected, type 1, and red AGN SEDs cover largely distinct regions in these figures. Optically extended sources tend to be IR bright for their X-ray flux and mostly lie within the range of the red AGN SEDs, implying the presence of an AGN that is not visible in the optical.

AGNs. Stronger IR flux, due for example to larger amounts of heated dust, may also contribute.

There is good separation of the type 1 and red AGN SED tracks in Figure 8. It is clearer than in Figure 7 that the majority of sources lie in the red AGN region, demonstrating the wider range of SED shapes and, in particular, the large population of red AGN in this hard X-ray-selected sample. Many of the extended, optically bright sources that were below the AGN region in Figure 7, lie within the red AGN SED region. This provides further evidence that they are predominantly obscured AGNs for which the optical colors are brightened by host-galaxy and/or polarized light contributions (Kuraszkiewicz et al. 2009a; Smith et al. 2003).

Moving to longer wavelength bands, the X-ray fainter sources are preferentially lost as the relatively higher flux limits cut down on the range of observable IR fluxes. This is most noticeable at $24 \mu \mathrm{m}$ (Figure 9) where the left-hand plot shows the $24 \mu \mathrm{m}$ versus the broadband X-ray flux and less than half the sample (333) are detected in the IR. The labeling is the same as for Figure 8.

\subsection{X-ray Sources Extended in Optical}

Of the 775 X-ray sources in the SWIRE/Chandra field, 107 were flagged as optically extended by the source extraction software (CASU pipeline, Irwin \& Lewis 2001). Visual inspection of the optical identifications of all the X-ray sources led to inclusion of an additional nine optically extended sources, and exclusion of 22 sources for false positives arising from three causes: spikes from nearby saturated stars; multiple closely spaced sources detected as a single, extended source; and proximity to a different, 

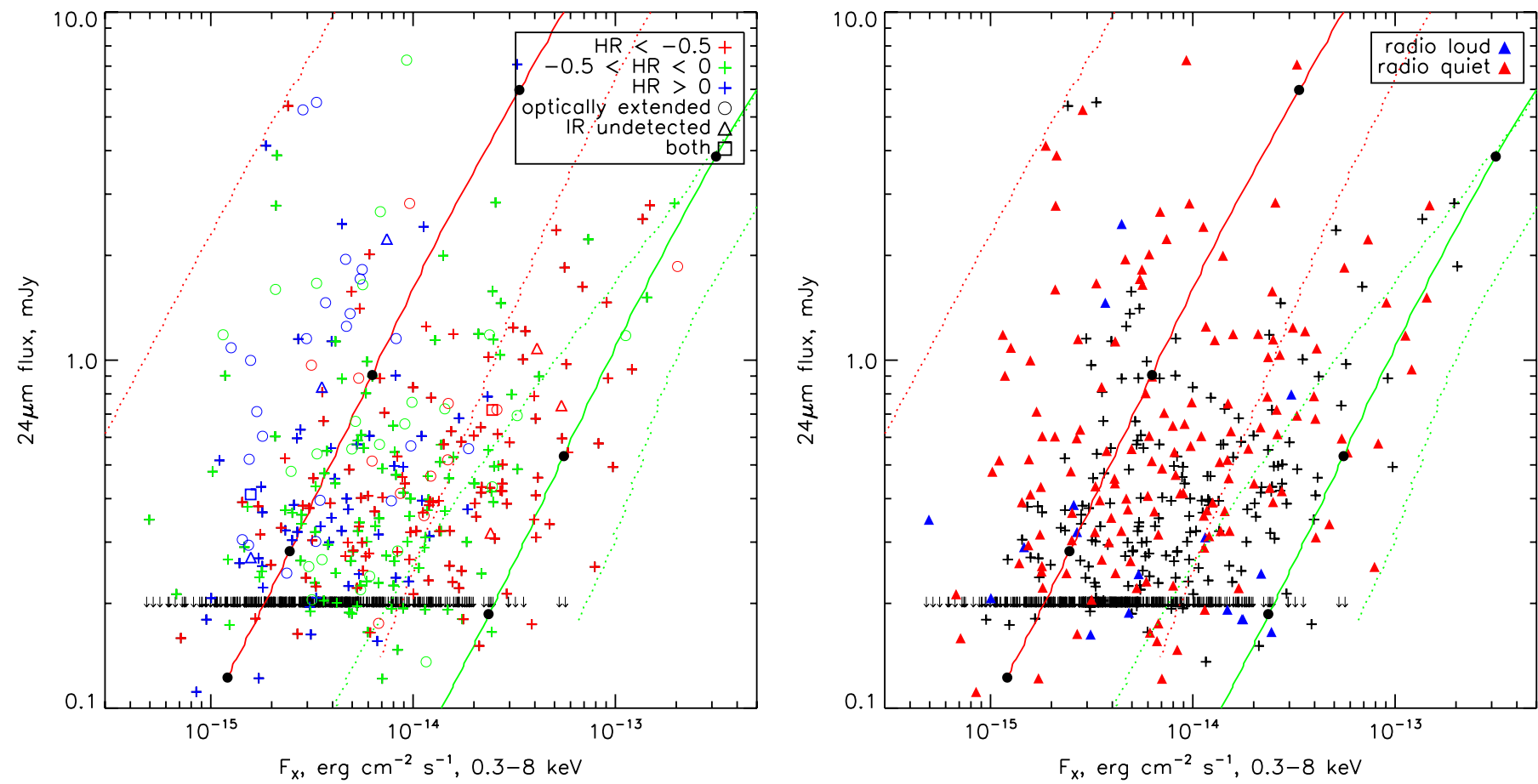

Figure 9. IR $24 \mu \mathrm{m}$ as a function of broadband X-ray flux for the SWIRE X-ray sources. Upper limits $(5 \sigma)$ are indicated by black arrows. Left: symbols and lines are as in Figure 7; right: sources are labeled according to their $q_{24}$ radio-loudness (Section 5.5.2).

unrelated extended source. A further two sources were excluded as it was determined that they are background AGNs coincident with the extended region of foreground galaxies. The verified list thus consists of 92 optically extended sources.

In order to determine some of the general properties of the sample, we derive luminosities from spectroscopic redshifts where available (29 of 92 sources; M. Polletta et al. 2010, in preparation) and from photometric redshifts for all but two of the remaining sources, which were too faint to obtain accurate photometry. Out of these 92 sources, 12 were identified as candidate non-nuclear ultraluminous X-ray sources (ULXs) based upon their positions within the host galaxies. Out of these 12 , nine have luminosities in excess of $10^{41} \mathrm{erg} \mathrm{s}^{-1}$ and are thus likely not individual X-ray binaries. A further five sources have luminosities below $10^{41} \mathrm{erg} \mathrm{s}^{-1}$ and are thus also considered ULX candidates, for a total of eight. These sources will be discussed in more detail in an upcoming paper (R. Kilgard et al. 2010, in preparation).

Most of the optically extended sources are at the optically bright edge or below the AGN region, where galaxies are expected to lie. Several of those within the AGN range are Sy2 galaxies. We summarize the properties of two unusual sources here.

CXOSW J104335.7+585249 has an SED that fits a Sy 1.8 template (Polletta et al. 2006) with a redshift $z \sim 0.5$. In the optical, it is a bright, complex galaxy. Its $\mathrm{X}$-ray flux is quite bright $\left(F(0.3-8 \mathrm{keV})=3.3 \times 10^{-15} \mathrm{erg} \mathrm{cm}^{-2} \mathrm{~s}^{-1}\right)$, with a hardness ratio $\sim 0$ implying moderate absorption, $N_{\mathrm{H}} \sim 10^{22} \mathrm{~cm}^{-2}$, lower than is usual for type 1.8-2 AGNs.

CXOSW J104552.4+590036 is faint and somewhat fuzzy in the optical and fits to a reddened AGN, MKN231-like SED (Polletta et al. 2006) at estimated redshift, $z \sim 2$ 2.8. Its X-ray hardness ratio, $\mathrm{HR} \sim-0.4$, implies little absorption. It has two nearby sources at 3.6 and $4.5 \mu \mathrm{m}$ that may affect these flux estimates.

\subsection{Radio-loudness}

It is common practice to divide AGNs into two classes based on the relative strength of their radio emission. However, there remain major questions concerning the reality of this dichotomy, its cause, and even the observational classification of radio-loud versus radio-quiet AGNs. For the former, physical properties such as larger central black hole mass (Lacy et al. 2001; Boroson 2002) and/or more rapid black hole spin in radio-loud sources have been suggested to cause either a dichotomy or a progression between one class and the other.

From an observational standpoint, radio-loudness is a useful tool to select out those sources with the strongest radio emission, either having large, extended structures or core-dominated and beamed. There are two alternative methods for defining a radio-loud source. First, using the radio-loudness, $R_{L}$, traditionally measured as $\log$ of the ratio of $6 \mathrm{~cm}$ radio to optical flux ( $B$ magnitude; Wilkes \& Elvis 1987; Kellermann et al. 1989 ), where values $>10$ indicate a radio-loud source. Second, using the absolute radio power where the number is around $\log L_{20 \mathrm{~cm}} \sim 31.6 \mathrm{erg} \mathrm{s}^{-1} \mathrm{~Hz}^{-1}$ (e.g. Zamfir et al. 2008). Since we do not have good redshift estimates for the full sample, we use the former definition here.

The bi-modality of the distribution of radio-loudness in AGNs is more/less pronounced depending on the sample selection (Kellermann et al. 1989; White et al. 2000; Lacy et al. 2001; Komossa et al. 2006; Zamfir et al. 2008). In addition, the use of the optical magnitude is questionable given that it is strongly effected by reddening and/or orientation (White et al. 2007), which are important constituents in IR/X-ray-selected samples (Kuraszkiewicz et al. 2009b). Figure 10 shows the distribution of radio-loudness $\left(R_{L}=\log \left[f_{20 \mathrm{~cm}} / f_{g^{\prime}}\right]\right)$ for the radio-detected subset of the current X-ray selected sample. The use of $g^{\prime}$ in place of $B$ magnitude has a negligible effect on the parameter given the small change in effective wavelength $(4770 \AA$ cf. 


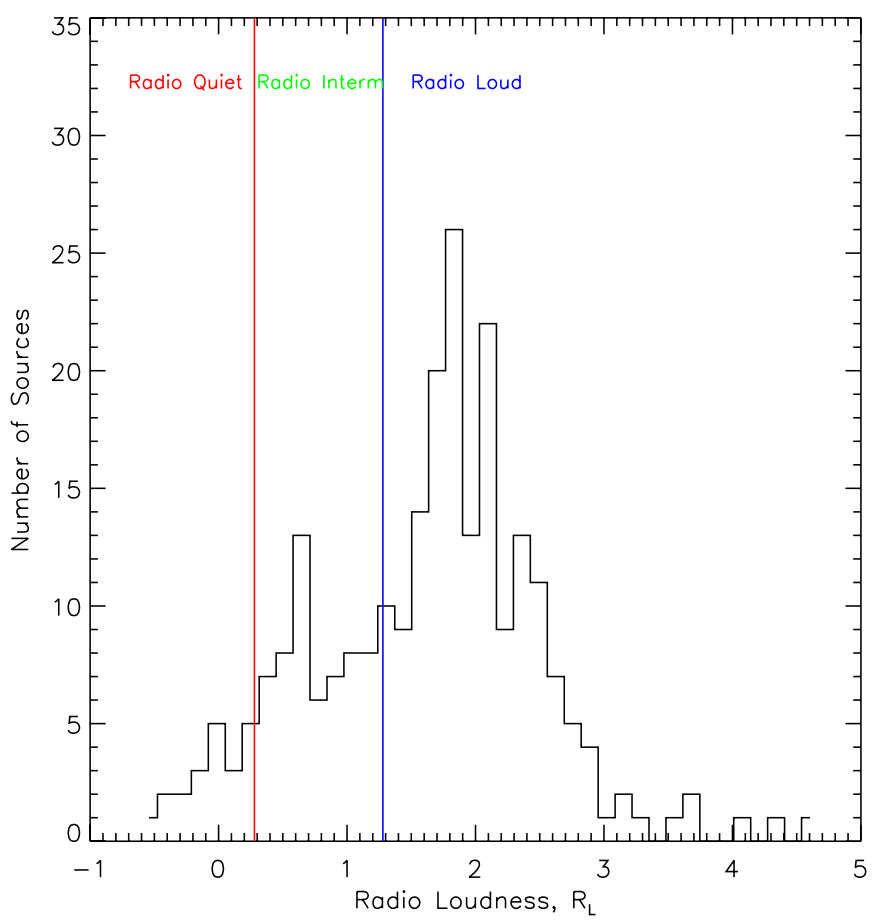

Figure 10. Histogram of the traditional measure of radio-loudness $\left(R_{L}=\right.$ $\left.\log \left[f_{20} \mathrm{~cm} / f_{g^{\prime}}\right]\right)$ for all radio and optically detected sources in the sample. The divisions between radio-loud $\left(R_{L}>19\right)$, radio-intermediate $\left(1.9<R_{L}<19\right)$, and radio-quiet $\left(R_{L}<1.9\right)$ for this data set are shown by vertical blue and red lines, respectively (Section 5.5)

$4400 \AA$ A; Fukugita et al. 1996). The use of $20 \mathrm{~cm}$ rather than $6 \mathrm{~cm}$ radio flux is corrected by assuming a spectral slope of 0.5 (where $f_{v} \propto v^{-\alpha}$ ) in the radio. This shifts the standard division between radio-loud (RL) and radio-quiet (RQ) from $R_{L}=10$ to $R_{L}=19$. Within our radio-detected subset, 74 sources have no detection at optical $g^{\prime}$ but all of these are classified as radio-loud based on the optical upper limit. The presence of beaming in the radio and/or the relative strength of the big blue bump, which is likely related to the accretion properties and/or Eddington ratio, affect radio-loudness so that a simple classification scheme does not ensure physically distinct sets of sources (Falcke et al. 1996). In addition, this X-ray-selected sample includes a larger number of sources whose optical flux is faint in comparison to optically selected, type 1 AGNs. To allow for the possible misclassification of sources around the traditional boundary, we divide the radio-quiet sources into an intermediate class (RI): $1.9<R_{L}<19$; and radio-quiet (RQ): $R_{L}<1.0$, as labeled in Figure 10, again following Kellermann et al. (1989) but noting that the division point is arbitrary. The $R_{L}$ distribution for this sample is not bi-modal, so there is no obvious division between radio-loud and radio-quiet sources. The distribution appears asymmetric, has a large radio-loud fraction using this traditional classification, and has a long tail toward radio-quiet sources. Of the 251 radio-detected X-ray sources in the VLA field, 174 are radio-loud, 60 radio-intermediate, and 17 radioquiet. Conservatively assuming that the non-radio-detected X-ray sources (317) are radio-quiet, ${ }^{17}>41 \%$ of this sample is classified as radio-loud using the traditional definition. This is much higher than the $\sim 5 \%-10 \%$ of radio-loud, broad-lined AGNs typically found in optical surveys (e.g., Peterson 1997). Few of the radio-loud objects are strong radio sources, such as would be found in radio catalogs. There are very few in the $R_{L}$ range of the radio-quiet AGNs in, e.g., the optically selected PG AGN (Kellermann et al. 1989).

\subsubsection{Radio and $X$-ray Properties}

In Figure 11 (left), we repeat Figure 7 but with the radio classes indicated. The radio-loud sources lie preferentially within the AGN region; those sources with low optical and

\footnotetext{
${ }^{17}$ We note that this is unlikely. The upper limits for more than half of these sources allow for them to be radio-loud. Since the radio flux limit is a strong function of position in the VLA field, those toward the field edge are even more likely to be radio-loud.
}
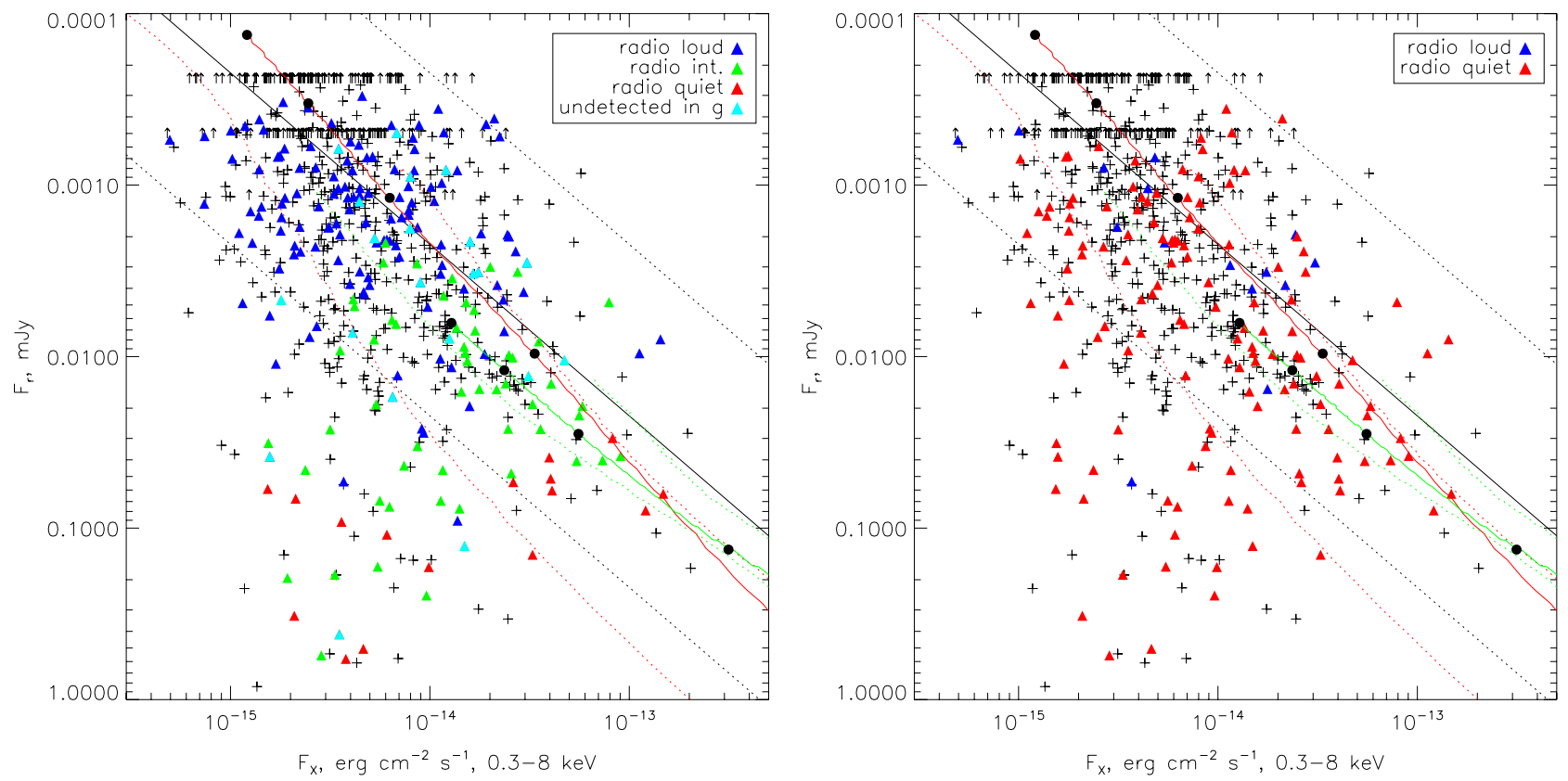

Figure 11. Optical $r^{\prime}$ band as a function of broadband X-ray flux for the SWIRE X-ray sources with sources labeled according to their radio class: left: using the traditional radio-loudness $\left(R_{L}\right)$ parameter (Figure 10); right: using $q_{24}>0.24$, to define the radio-loud AGN. Other lines and symbols are as in Figure 7. The large number of radio loud sources as defined by $R_{L}\left(\right.$ left), lying at faint optical fluxes in the red AGN region, are mostly classified as radio-quiet when the more stable $q_{24}$ classification method is used to define radio-loud (right). 

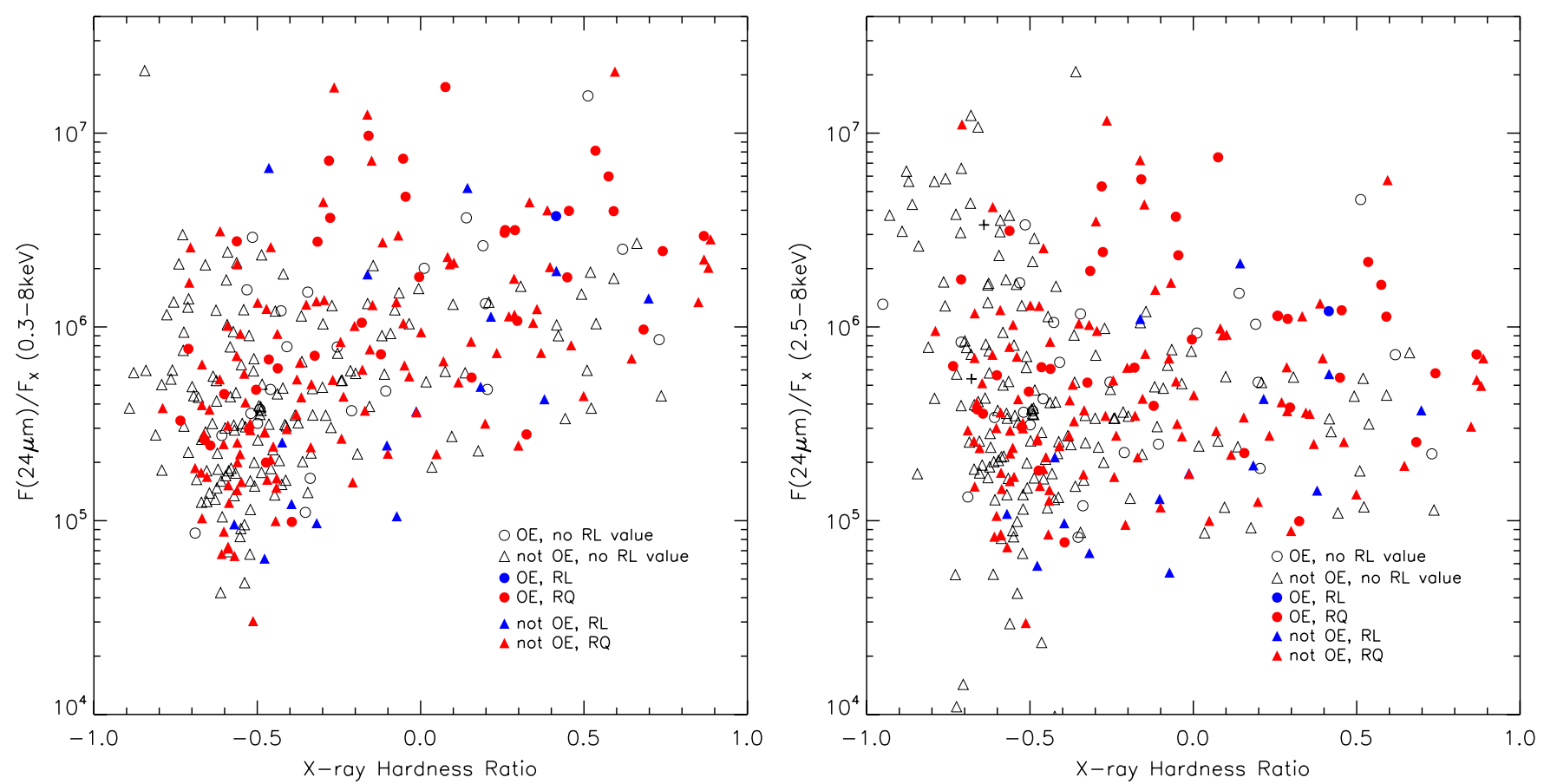

Figure 12. Dimensionless ratio of $24 \mu \mathrm{m}$ to effective broad (left) and hard (right) X-ray flux density (determined in $\mu \mathrm{Jy}$ at $1 \mathrm{keV}$ from the hard and broadband fluxes respectively, assuming $\Gamma=1.7$ ) as a function of X-ray hardness ratio for those X-ray sources with $24 \mu \mathrm{m}$ identifications. The tendency for hard sources to have higher $24 \mu \mathrm{m}$ to X-ray flux ratios is present only for broadband X-ray flux, indicating that this is an absorption effect in the X-ray alone. The colors indicate the $q_{24}$ radio class (Section 5.5.2). Symbols are defined in the legend, where OE denotes optically extended.

X-ray flux are almost exclusively radio-loud. This is partially due to the fact that all radio-detected sources with fluxes close to the optical and/or X-ray flux limits will be classified as radioloud because the transition between radio-loud and radio-quiet classes falls below the faintest radio flux limit. Those that are radio-undetected cannot be classified.

However, we must also examine the meaning of radio-loud in this case. As discussed in Section 5.1, the red and green lines in Figure 11 indicate the regions in which AGNs with red SEDs and those with SEDs typical of optically selected, type 1 AGNs (respectively) lie as a function of redshift. The sources at the optical and X-ray faint end lie beyond the range of optically selected, type 1 AGN SEDs (green line) but within the range of the red SEDs (red line). We deduce that they are relatively optically faint (as shown in Kuraszkiewicz et al. $2009 b$ ). As noted above, if the optical emission is relatively faint while the radio is unaffected, the radio-loudness parameter, $R_{L}$, breaks down as an indicator of bright radio emission. Thus an abnormally large fraction of the sample is incorrectly classified as radio-loud, as is clear in Figure 11. While $R_{L}$ works well for optically selected, type 1, blue AGNs, an alternative radioloudness indicator is needed for the remainder of the AGN population.

\subsubsection{Radio-loudness Determined Using $24 \mu \mathrm{m}$ Flux, $q_{24}$}

Given the limitations of $R_{L}$, it has been suggested that IR fluxes provide a more stable measure of the radio-loudness. In particular, $q_{24}\left(=\log \left(f_{24 \mu \mathrm{m}} / f_{20} \mathrm{~cm}\right)\right.$; Appleton et al. 2004; Kuraszkiewicz et al. 2010) is minimally effected by Eddington ratio, host-galaxy contribution, redshift and/or reddening. The stability of the $24 \mu \mathrm{m}$ flux to obscuration is shown in Figure 12 where the ratio of the $24 \mu \mathrm{m}$ flux to the broad (left) and hard (right) band X-ray fluxes is plotted as a function of $\mathrm{X}$-ray hardness ratio. The broadband X-ray flux shows that hard,

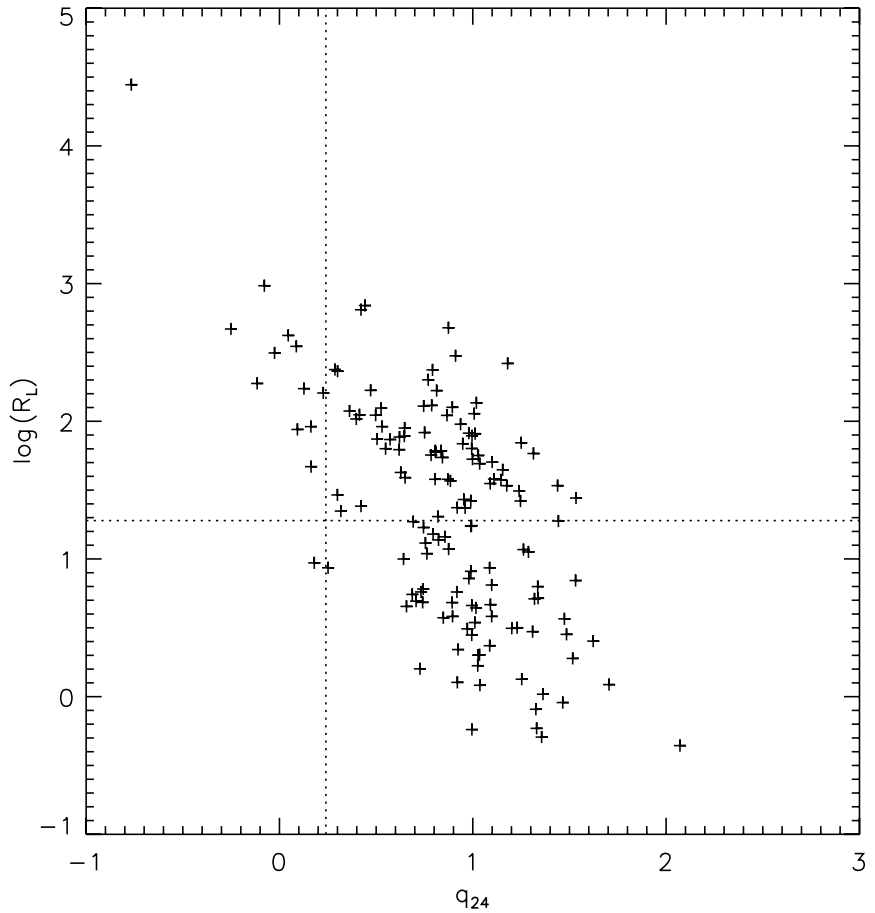

Figure 13. Radio-loudness $\left(R_{L}\right)$ as a function of mid-IR-to-radio flux ratio $\left(q_{24}\right)$. The radio-loud/quiet boundaries for each parameter $\left(R_{L}=19, q_{24}=0.24 \pm\right.$ 0.12 ), the latter based on a comparison using a large sample of type 1 quasars (Kuraszkiewicz et al. 2010), are marked with dashed lines. The large number of crossover sources in our X-ray-selected sample are visible in the top right quadrant.

low-redshift sources have higher $24 \mu \mathrm{m}$ flux relative to the $\mathrm{X}$-ray while the hard-band X-ray flux does not. The effect is most likely due to absorption in the X-ray band and indicates 

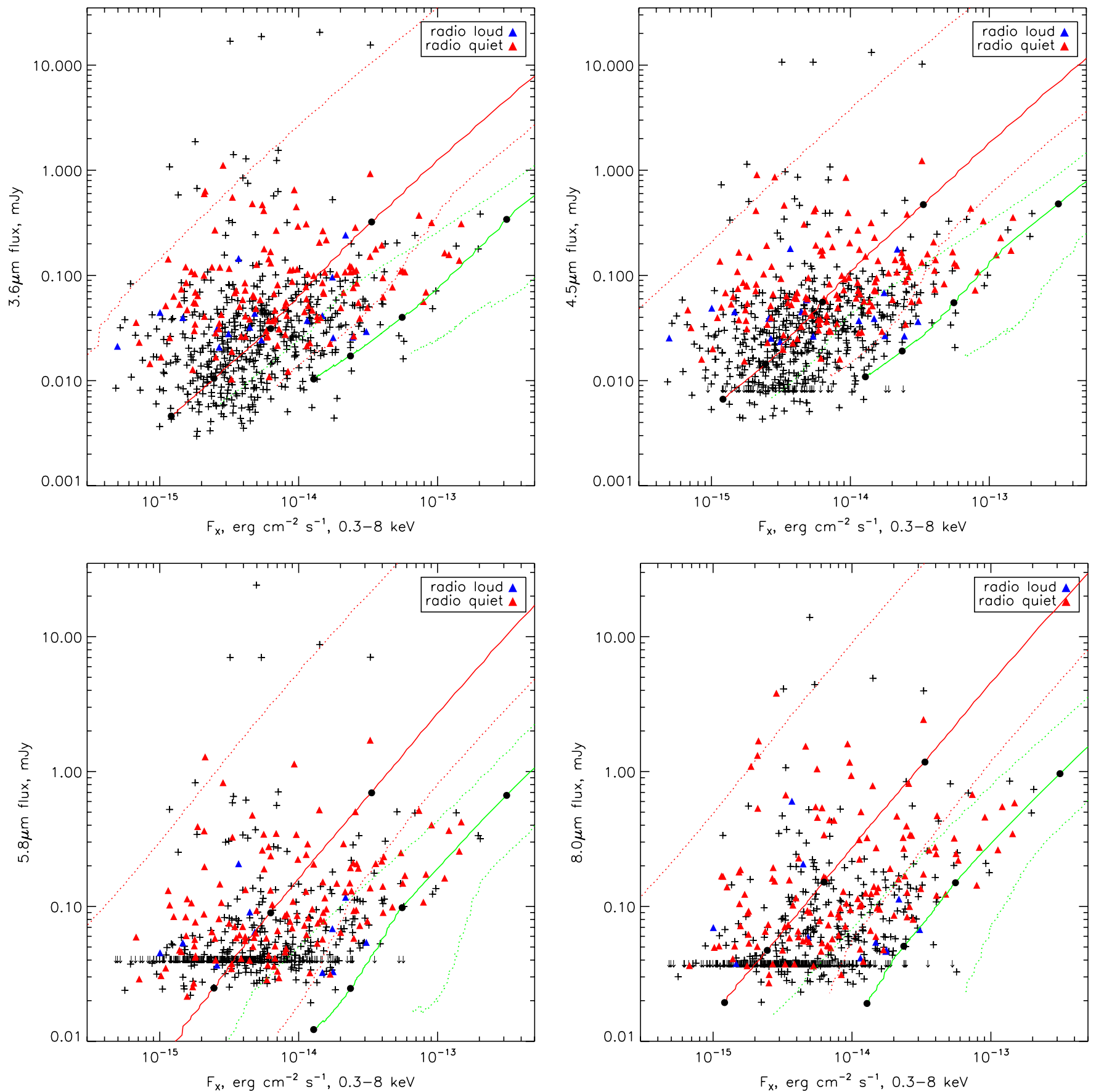

Figure 14. Spitzer IR as a function of broadband X-ray flux for the SWIRE X-ray sources in each IRAC band. Symbols are as in Figure 8, but with radio class defined using $q_{24}$.

that the $24 \mu \mathrm{m}$ flux is not affected by the X-ray absorbing material.

In Figure 13, we show the relation between $q_{24}$ and $R_{L}$ for the 147 sources in our sample for which data are available in all bands. The majority of the sources are distributed around $q_{24} \sim 1$, consistent with the IR-radio correlation for galaxies, on which radio-quiet AGNs also lie (Appleton et al. 2004). Kuraszkiewicz et al. (2010) make a systematic comparison of the two parameters for a large sample of blue, type 1 quasars selected from the WSRT radio survey. The historically used transition from radio-loud to radio-quiet, $R_{L}>19$, for the current data set and the equivalent value for $q_{24}(=0.24 \pm$
0.12) derived by Kuraszkiewicz et al. (2010) are indicated by dotted lines. This figure dramatically shows the large number of cross-over sources, those in the top right-hand quadrant, which are radio-loud according to $R_{L}$ and radio-quiet according to $q_{24}$. The conclusion that radio classification using $R_{L}$ is incorrect for these AGNs is reinforced by the fact that they have $q_{24}$ $\sim 1$, as expected for radio-quiet AGNs and galaxies (Appleton et al. 2004). Using $q_{24}, 13$ of the 147 sources are radio-loud, in excellent agreement with the expected 5\%-10\% of radioloud AGNs based on optically selected, type 1 (unobscured, blue) AGNs (Peterson 1997). Figure 11 (right-hand side) shows the same plot as the left-hand side but using $q_{24}$ to define 
radio-loudness. A much smaller fraction of sources is radioloud, and they are not systematically placed in the figure.

Figure 14 shows the Spitzer IRAC fluxes as a function of $\mathrm{X}$-ray flux (as in Figure 8) with $q_{24}$-classified, radio-loud sources indicated. There is no visible relation between the radio class and the relative IR and X-ray fluxes.

We conclude that $q_{24}$ is far superior to $R_{L}$ for classification of radio-loud versus radio-quiet AGNs.

\section{CONCLUSIONS}

We present a list of 775 Chandra X-ray sources in the SWIRE/Chandra medium-depth, X-ray survey. Cross correlation with Spitzer, optical, and radio images of part/all of the fields resulted in $771(99 \%)$ identifications in at least one optical/IR band, 767 have IR counterparts visible in the Spitzer data in at least one (IRAC) band, and 333 have $24 \mu \mathrm{m}$ with MIPS detections. Four of the sources have no optical/IR counterpart down to our flux limits, and four have an optical but no IR counterpart. We present multi-wavelength flux measurements for 744 $\mathrm{X}$-ray sources, all those which are uncontaminated, unconfused, and above the formal survey thresholds. The near-IR-X-ray data sets are well matched in the flux limit and go deep into the AGN population, providing an excellent data set for multi-wavelength studies of the full AGN population, which will be reported in a companion paper (M. Polletta et al. 2010, in preparation). As in earlier surveys (DK04), there is no correlation between X-ray hardness and hard X-ray flux in this sample, confirming that hardness is predominantly caused by obscuration in the X-rays.

The very deep (2.7 $\mu \mathrm{Jy}$ at the field center) VLA data, covering part of the Chandra field (Figure 1), results in 251 ( $>4 \sigma$ ) radio detections, $44 \%$ of the $568 \mathrm{X}$-ray sources in the VLA field. Even the very deepest radio data cannot detect all the X-ray sources. We demonstrate that the traditional radio-to-optical flux ratio, $R_{L}$, used to define radio-loudness in the AGN breaks down for a large proportion of the sources in this X-ray-selected sample due to the weakness of the optical emission. Use of the $24 \mu \mathrm{m}$ flux in place of the optical, $q_{24}$ parameter (Appleton et al. 2004), brings the radio-loud fraction down to expected levels (9\%) and is strongly preferred as an indicator of radio-loudness for the full AGN population.

The wide range of optical/IR/X-ray SEDs in this X-rayselected sample is demonstrated by the lack of any correlation between the optical or IR flux and the X-ray flux. Comparison with tracks for optically selected, type 1, and red AGN SEDs demonstrates the predominance of red AGNs in this sample. There is a continuous distribution rather than distinct classes of AGNs, and the IR versus X-ray plots allow better discrimination than the optical as the effects of obscuration are much lower. Comparison of the source properties (X-ray hardness, flux ratios, and radio loudness) with the predictions of the standard median SEDs allows us to broadly classify the source types as a function of position in flux-flux plots. A correlation between the $24 \mu \mathrm{m}$ flux and the X-ray hardness disappears when the hard X-ray flux is used, demonstrating that it is due to absorption in the X-rays while the $24 \mu \mathrm{m}$ flux remains unaffected. This reinforces the use of $q_{24}$ to define radio-loudness due to its stability.

This work is based in part on observations made with the Spitzer Space Telescope, which is operated by the Jet Propulsion Laboratory, California Institute of Technology under a contract with NASA. We gratefully acknowledge the financial support of NASA Contract: NAS8-03060 (Chandra X-ray Center, B.W.) and Chandra GO grant: GO4-5158A (R.K.). M.P. acknowledges financial contribution from contracts ASI-INAF I/016/07/0 and ASI-INAF I/088/06/0.

\section{REFERENCES}

Alexander, D. M., Vignali, C., Bauer, F. E., Brandt, W. N., Hornschemeier, A. E., Garmire, G. P., \& Schneider, D. P. 2002, AJ, 123, 1149

Alexander, D. M., et al. 2003, AJ, 123, 539

Antonucci, R. R. J., \& Miller, J. S. 1985, ApJ, 297, 621

Appleton, P. N., et al. 2004, ApJS, 154, 147

Barmby, P., et al. 2006, ApJ, 642, 126

Basilakos, S., Plionis, M., Georgakakis, A., \& Georgantopoulos, I. 2005, MNRAS, 356, 183

Boroson, T. A. 2002, ApJ, 565, 78

Brusa, M., et al. 2005, A\&A, 432, 69

Cardamone, C. N., et al. 2008, ApJ, 680, 130

Chiappetti, L., et al. 2005, A\&A, 439, 413

Comastri, A., et al. 2002, ApJ, 571, 771

Cutri, R. M., Nelson, B. O., Francis, P. J., \& Smith, P. S. 2002, in ASP Conf. Ser. 284, AGN Surveys, Proc. IAU Colloquium 184, ed. R.F. Green, E.Ye. Khachikian, \& D.B. Sanders (San Francisco, CA: ASP), 127

Daddi, E., et al. 2007, ApJ, 670, 173

Donley, J. L., Reike, G., Perez-Gonzalez, P. G., \& Barro, G. 2008, ApJ, 687, 111

Elvis, M. S., et al. 1994, ApJ, 95, 1

Elvis, M. S., et al. 2009, ApJS, 184, 158

Falcke, H., Sherwood, W., \& Patnaik, A. R. 1996, ApJ, 471, 106

Feruglio, C., et al. 2008, A\&A, 488, 417

Fiore, F., et al. 2008, ApJ, 672, 94

Franceschini, A., et al. 2005, AJ, 129, 2074

Fukugita, M., Ichikawa, T., Gunn, J. E., Doi, M., Shimasaku, K., \& Schneider, D. P. 1996, AJ, 111, 1748

Gehrels, N. 1986, ApJ, 303, 336

Gilli, R., Comastri, A., \& Hasinger, G. 2007, A\&A, 463, 79

Gorjian, V., et al. 2008, ApJ, 679, 1040

Hickox, R., et al. 2009, ApJ, 696, 891

Hopkins, P. F., Hickox, R., Quartet, E., \& Hernquist, L. 2009, MNRAS, 398, 333

Irwin, M., \& Lewis, J. 2001, New Astron. Rev., 45, 105

Kellermann, K. I., Sramek, R., Schmidt, M., Shaffer, D. B., \& Green, R. 1989, AJ, 98, 1195

Kim, D.-W., \& Fabbiano, G. 2003, ApJ, 586, 826

Kim, D.-W., et al. 2004, ApJS, 150, 19 (DK04)

Kim, M., Wilkes, B. J., Kim, D.-W., Green, P. J., Barkhouse, W. A., Lee, M. G., Silverman, J. D., \& Tananbaum, H. D. 2007b, ApJ, 659, 29

Kim, M., et al. 2007a, ApJS, 169, 401 (MK07)

Komossa, S., Voges, W., Dawei, A., Mathur, S., Adorf, H-M., Lemson, G., Duschl, W., \& Grupe, D. 2006, AJ, 132, 531

Kuraszkiewicz, J., Wilkes, B. J., Schmidt, G., Smith, P. S., Cutri, R., \& Czerny, B. 2009a, ApJ, 692, 1180

Kuraszkiewicz, J., et al. 2009b, ApJ, 692, 1143

Kuraszkiewicz, J., et al. 2010, ApJ, submitted

Lacy, M., Laurent-Muehleison, S. A., Ridgeway, S. E., Becker, R. H., \& White, R. L. 2001, ApJ, 551, 17

Laird, E. S., et al. 2009, ApJS, 180, 102

Lehmer, B. D., et al. 2005, ApJS, 161, 21

Luo, B., et al. 2008, ApJS, 179, 19

Norman, C., et al. 2002, ApJ, 571, 218

Owen, F. N., \& Morrison, G. E. 2008, ApJ, 136, 1889

Park, S. Q., Kashyap, V. L., Siemiginowska, A., van Dyk, D. A., Zezas, A., Heinke, C., \& Wargelin, B. 2006, ApJ, 652, 610

Park, S. Q., et al. 2008, ApJ, 678, 744

Peterson, B. M. 1997, An Introduction to Active Galactic Nuclei (Cambridge: Cambridge Univ. Press)

Polletta, M. C., et al. 2006, ApJ, 642, 673

Polletta, M., et al. 2007, ApJ, 663, 81

Sambruna, R. M., Reeves, J. N., \& Braito, V. 2007, ApJ, 665, 1030

Schlegel, D. J., Finkbeiner, D. P., \& Davis, M. 1998, ApJ, 500, 525

Shupe, D. L., et al. 2008, AJ, 135, 1050

Smith, P. S., Schmidt, G. D., Hines, D. C., \& Foltz, C. B. 2003, ApJ, 593, 676

Surace, J. 2005, SWIRE Data Delivery Document II, Tech. Rep., Spitzer Science Center (Pasadena, CA: Cal. Tech.)

Stark, A. A., Gammie, C. F., Wilson, R. W., Bally, J., Linke, R. A., Heiles, C., \& Hurwitz, M. 1992, ApJS, 79, 77

Stocke, John T., et al. 1991, ApJS, 76, 813

Tozzi, P., et al. 2006, A\&A, 451, 457 
Trouille, L., Barger, A. J., Cowie, L. L., Yang, Y., \& Mushotzky, R. F. 2008, ApJS, 179, 1

Treister, E., Urry, C. M., \& Virani, S. 2009, ApJ, 696, 110

Treister, E., et al. 2004, ApJ, 616, 123

Vikhlinin, A., Forman, W., Jones, C., \& Murray, S. S. 1995, ApJ, 451, 542

White, R. L., Helfand, D. J., Becker, R. H., Glikman, E., \& de Vries, W. 2007, ApJ, 654, 99
White, R. L., et al. 2000, ApJS, 126, 133

Wilkes, B. J., \& Elvis, M. S. 1987, ApJ, 323, 243

Witt, H. J., Czerny, B., \& Zycki, P. T. 1997, MNRAS, 286, 848

Yang, Y., Mushotzky, R. F., Steffan, A. T., Barger, A. J., \& Cowie, L. L. 2004, AJ, 128,1501

Zamfir, S., Sulentic, J. W., \& Marziani, P. 2008, MNRAS, 387, 856 